\title{
Structure-based level set method for automatic retinal vasculature segmentation
}

\author{
Bekir Dizdaroğlu ${ }^{1,2^{*}}$, Esra Ataer-Cansizoglu² ${ }^{2}$ Jayashree Kalpathy-Cramer ${ }^{3}$, Katie Keck ${ }^{4}$, Michael F Chiang ${ }^{4,5}$ \\ and Deniz Erdogmus ${ }^{2}$
}

\begin{abstract}
Segmentation of vasculature in retinal fundus image by level set methods employing classical edge detection methodologies is a tedious task. In this study, a revised level set-based retinal vasculature segmentation approach is proposed. During preprocessing, intensity inhomogeneity on the green channel of input image is corrected by utilizing all image channels, generating more efficient results compared to methods utilizing only one (green) channel. A structure-based level set method employing a modified phase map is introduced to obtain accurate skeletonization and segmentation of the retinal vasculature. The seed points around vessels are selected and the level sets are initialized automatically. Furthermore, the proposed method introduces an improved zero-level contour regularization term which is more appropriate than the ones introduced by other methods for vasculature structures. We conducted the experiments on our own dataset, as well as two publicly available datasets. The results show that the proposed method segments retinal vessels accurately and its performance is comparable to state-of-the-art supervised/unsupervised segmentation techniques.
\end{abstract}

Keywords: Color retinal fundus images; Phase map; Segmentation of retinal vasculature; Structure and texture parts of retinal fundus image; Structure-based level set method

\section{Introduction}

Published ophthalmology studies reveal that there are often significant differences in clinical diagnosis of retinal diseases among medical experts [1]. Some of these approaches involve tedious processes. Manual segmentation has become more and more time consuming with the increasing amount of patient data. An automatic retinal vasculature segmentation method may become an integral part of a computer-based image analysis and diagnosis systems with improved accuracy and consistency [2].

Considering the conducted research, literature is full of examples [3-10] on vasculature segmentation, detection, and other kinds of analysis employing especially supervised/unsupervised classification of pixels in retinal fundus images [11-19]. Marin et al. [14] and Soares et al. [15] presented two different supervised methods for segmentation of retinal vasculature by using moment

\footnotetext{
*Correspondence: bekir@ktu.edu.tr

${ }^{1}$ Department of Computer Engineering, Karadeniz Technical University,

Trabzon 61080, Turkey

${ }^{2}$ Cognitive Systems Laboratory, Northeastern University, Boston, MA 02115 , USA

Full list of author information is available at the end of the article
}

invariant-based features and 2-D Gabor filters, respectively. Staal et al. [16] proposed a retinal vasculature segmentation method using centerlines of a vessel base that are extracted by using image ridges. Budai et al. [17] presented an improved approach using Frangi's method [18]. Other studies have employed centerline tracing methods and principal curves $[19,20]$. The reader may refer to [21] for more related studies in the literature.

Level set-based methods have been widely used for image segmentation [22-34]. In general, these methods can be classified under two categories: (i) edge-based [22-30] and (ii) region-based [31-34] methods. However, level set-based methods have not been extensively employed in retinal vasculature segmentation. To the best of our knowledge, there have been only a few studies in the literature proposing methods based on level sets to trace vasculature in retinal fundus images. This is due to challenges of vessel shapes in level set-based image segmentation methods [24]. Major challenges posed by the very thin and elongated structure of retinal vessels are further compounded by poor contrast in regions of interest for level set-based segmentation 
methods. In one of those studies [24], the level set-based method is applied only on a selected region of images by implementing a non-automatic initialization of zero-level contours. These regions do not have any non-uniform intensity values. The method in [24] also employs edge information based on phase map and uses a reinitialization process to regularize the level set function, which is a problem in level set-based framework [25]. Moreover, this process requires complex discretization especially for re-initialization of the level set function. In addition, the method employs fixed filter coefficients to generate image features such as edges by using the $\log$-Gabor filter, which does not generate a proper output to trace extremely thin retinal vessels in fundus images smoothly. The level set segmentation method [26] proposed by Pang et al. requires the selection of initial contour in the form of long strips in the vertical direction, and this is not an optimal selection. This selection leads to an increase in the number of iterations to generate the results. According to the accuracy metric, the method produces poor results quantitatively on a nonpathological fundus image. Although they claim to present a fully automated method, the system requires mask images from the user. There are other level set approaches [27-29,31-34] that focus on segmenting other vasculature structures in different image modalities such as ultrasound images and magnetic resonance images (MRIs). However, these region-based methods $[32,33]$ cannot be used extensively in segmentation of retinal fundus images due to the form of vascular structures. Another method presented for retinal vessel segmentation [34] employs region-based level sets and region growing approaches, simultaneously.

In this paper, we present an improved and automatic level set-based method for retinal vasculature segmentation. The presented method utilizes a robust phase map to determine image structures and seed points around the vessels in the initialization of the level set function. The performed tests on pathological and non-pathological fundus images demonstrate that the proposed method performs better than the existing approaches based on level sets.

The organization of the paper is structured as follows. 'Section 2' introduces the general information about retinal fundus images and level set-based methods developed for segmentation. 'Section 3' explains the proposed method and compares it with the existing approaches in the literature. Experimental results are given in 'Section 4.' Finally, 'Section 5' presents a conclusion and possible future work in the field.

\section{Background}

Let I: $\Omega \rightarrow \mathbb{R}^{3}$ be a color image defined on domain $\Omega \rightarrow$ $\mathbb{R}^{2}$, and let $I_{i}: \Omega \rightarrow \mathbb{R}$ represent the $i$ th color channel of the image I. Let $\mathbf{p}=(x, y) \in \Omega$, denote any point in $\Omega$. Digital images have two additive components: structure part and texture part. These can be visualized as the cartoon version with sharp edges and noisy/textured version of the original image, respectively [35-37].

\subsection{Characteristics of retinal fundus images}

Retinal fundus images can be generated in color or grayscale format in digital media. The pixels of a retinal fundus image are represented as color values in $R G B$ color space as seen in Figure 1a,b. In terms of representation of retinal vessels, these images have mostly structure information but also a texture part (noise, defects, etc.). The retinal fundus images can be split into two categories, namely the pathological retinal fundus images and the non-pathological ones. The aim of segmentation methods for retinal fundus images is to separate vasculatures from other regions as can be seen in Figure 1c,d. However, due to the structure of the optic disk and macula, segmentation of blood vessels of retinal images is difficult. These regions have a more prominent intensity inhomogeneity compared to other parts of retinal images. Furthermore, pathological images may contain defects and disorders such as drusen, geographic atrophy (GA), and non-uniform intensities. Such disorders also make the process of segmentation complicated.

As shown in Figure 2, each color channel in $R G B$ color space can be separated and treated as an independent grayscale image. Considering those channels, the green channel component of the retinal image gives the best structure information to be processed $[15,19]$ even though some regions such as the optic disk and macula in this channel component have non-uniform intensity levels. Let us use $I$ instead of $I_{2}$ to represent the green channel component of the given image. In this case, the model would be as in $I=b J+$ noise (defects) [33], where $b J$ and noise are considered as the structure component and the texture component, respectively. The green channel of the given image has some noises but no defects such as drusen, GA, etc.; the noise can be reduced using a convolution with a Gaussian filter $G_{\sigma}$ of standard deviation $\sigma$. In the above equation, $J$ is the true image, which consists of almost all constant values in an image region such as the optic disk, and $b$ is referred to as the intensity inhomogeneity (shading artifact), which changes slowly throughout that image region.

\subsection{Edge-based level set segmentation approach}

In this section, we give brief information about segmentation of object and background using edge-based level set methods. Let $C$ be a closed subset of $\Omega$, that is, the union of a finite set of smooth Jordan curves $C_{i}$. Let $\Omega_{i}$ be the connected regions of $\Omega \backslash C$ bounded by $C_{i}$. $C$ can be expressed as the zero-level contour of some scalar 


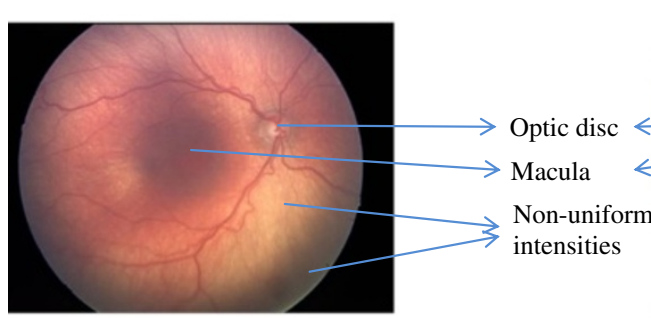

(a)

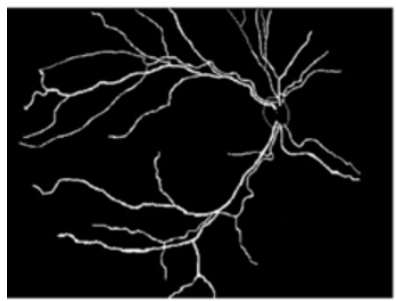

(c)

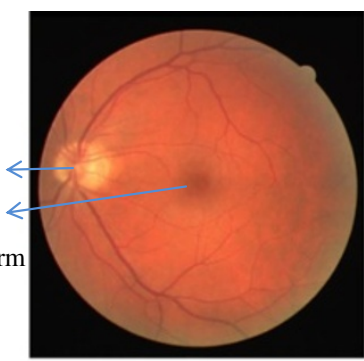

(b)

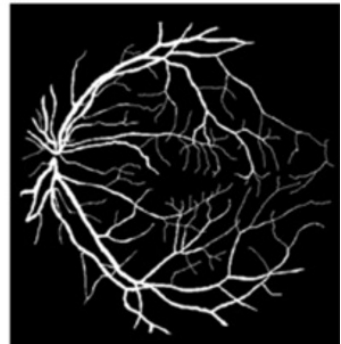

(d)

Figure 1 Sample retinal fundus images and manual segmentations. A pathological image (of size $640 \times 480$ pixels) from our own dataset $([2,19])$ given in (a), a non-pathological image (of size $565 \times 584$ pixels) from the DRIVE dataset ([3]) given in (b), the manual segmentation image of (a) given in (c), and the manual segmentation image of (b) given in (d).

Lipschitz continuous function $\Phi: \Omega \rightarrow \mathbb{R}$ [22]. The level set evolution equation of the curve $C$ with the speed function $F$ is as given in Equation 1:

$$
\frac{\partial \Phi}{\partial t}=F(\|\nabla \Phi\|)
$$

Iterations of level set evolution are adversely affected by numerical errors and other factors that cause irregularities. Therefore, a frequent re-initialization process, formulated as $\partial \Phi / \partial t=\operatorname{sign}\left(\Phi_{0}\right)(1-\|\nabla \Phi\|)$, could be included to restore the regularity of the level set function, establishing a stable level set evolution. Here, $\Phi_{0}$ is the level set function to be re-initialized and $\operatorname{sign}($. stands for signum function. Re-initialization is performed by interrupting the evolution periodically and correcting irregularities of the level set function using a signed distance function. Even with a re-initialization process, in most of the level set methods such as the geodesic active counters (GAC) model [23], irregularities can still emerge [25]. Therefore, $\mathrm{Li}$ et al. introduced a new energy term called level set function regularization [25].

Image segmentation based on level set methods typically consists of two additively combined energy terms, which are the length regularization term and the speed

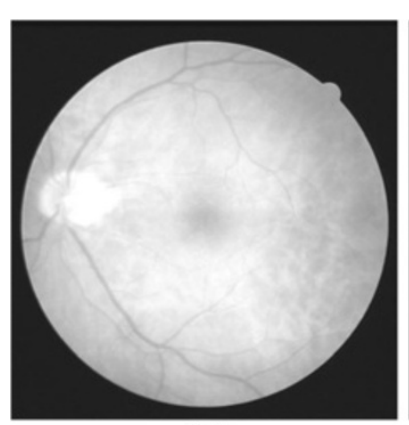

(a)

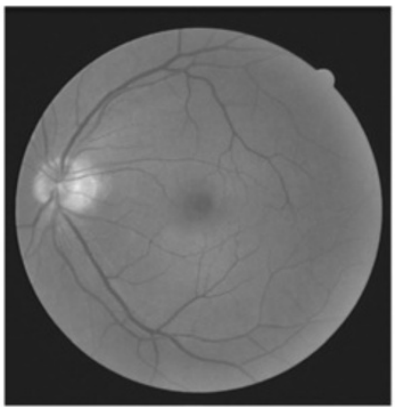

(b)

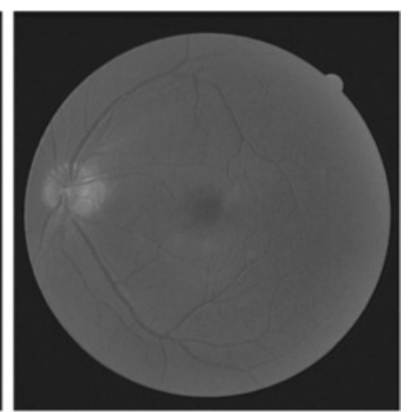

(c)

Figure 2 Color channel components of the non-pathological retinal fundus image presented in Figure 1b. Red (a), green (b), and blue (c) channel components. 
term related to the weighted area. The model is defined as $E(\Phi)=\mu R(\Phi)+\vartheta L(\Phi)+\alpha A(\Phi)$, where $R(),. L($.$) , and$ $A($.$) are the level set function regularization term, the$ zero-level contour regularization term, and the term adjusting the speed of motion to zero-level contour, respectively. Here, $\mu, \vartheta$, and $\alpha$ are weighting parameters.

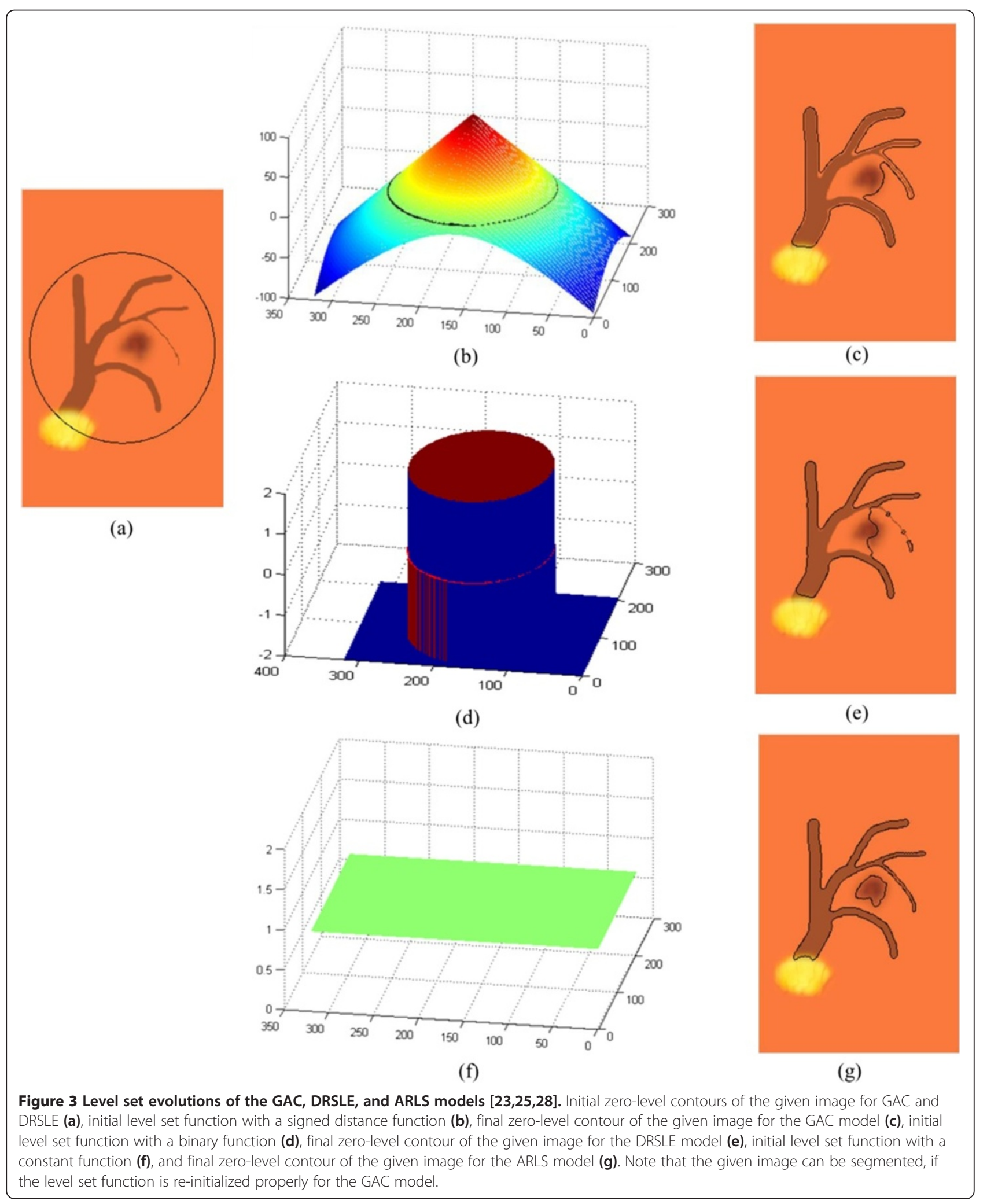


The level set function can be initialized in three different ways. In order to demonstrate the effect to the segmentation results, instead of a retinal fundus image, we employ a synthetic image that comprises artificially similar vessels and defects (Figure 3).

1. Initialization with a signed distance function, $d($. (GAC model [23]) (Figure 3a,b,c):

$$
\Phi_{\text {initial }}(\mathbf{p})=\left\{\begin{array}{lrl}
-d(\mathbf{p}, C) & \text { in } \Omega_{0} & \text { where } \Omega_{0} \text { (marked by the } \\
0 & \text { on } C & \text { user or selected automatically) } \\
d(\mathbf{p}, C) & \text { in } \Omega \backslash \Omega_{0} & \text { is an initial region in } \Omega .
\end{array}\right.
$$

2. Initialization with a binary function (distance regularized level set evolution (DRLSE) model [25]) (Figure 3a,d,e): $\Phi_{\text {initial }}=\left\{\begin{array}{ll}-c_{0} & \text { in } \Omega_{0} \\ c_{0} & \text { in } \Omega \backslash \Omega_{0}\end{array}\right.$, where $c_{0}$ is a small valued constant.

3. Initialization with a constant function (adaptive regularized level set (ARLS) model [28]) (Figure 3f,g): $\Phi_{\text {initial }}=\mp c_{0}$ in $\Omega$.

Edge-based level set methods have some drawbacks. Sometimes, a global minimum cannot be found and the methods tend to be slower than other segmentation methods. The global minimum can be correctly obtained if the initial contour is set properly. Level set-based methods also run faster when a narrow band approach is employed in the segmentation process.

\section{The proposed method}

Our method can be considered in three main steps as outlined in Figure 4:

\section{Preprocessing}

2. Modified phase map estimation

3. Structure-based level set segmentation

More details about these steps are given in the following subsections of 3.1, 3.2, and 3.3.

\subsection{Preprocessing for correction of non-uniform intensity}

A preprocessing step is employed for the correction of intensity inhomogeneity of retinal fundus images. Firstly, we apply a trace-based method to reduce noise and then a shock filter is applied to sharpen the image. Both filters work based on color information and give more robust results compared to the scalar approaches presented in $[19,38]$. Secondly, the green channel of the filtered image is extracted. Thirdly, two different images are generated by applying adaptive histogram equalization on the green channel image and then by applying a classical median filter on the equalized histogram image [19]. Lastly, depending on the case (intensity inhomogeneity),

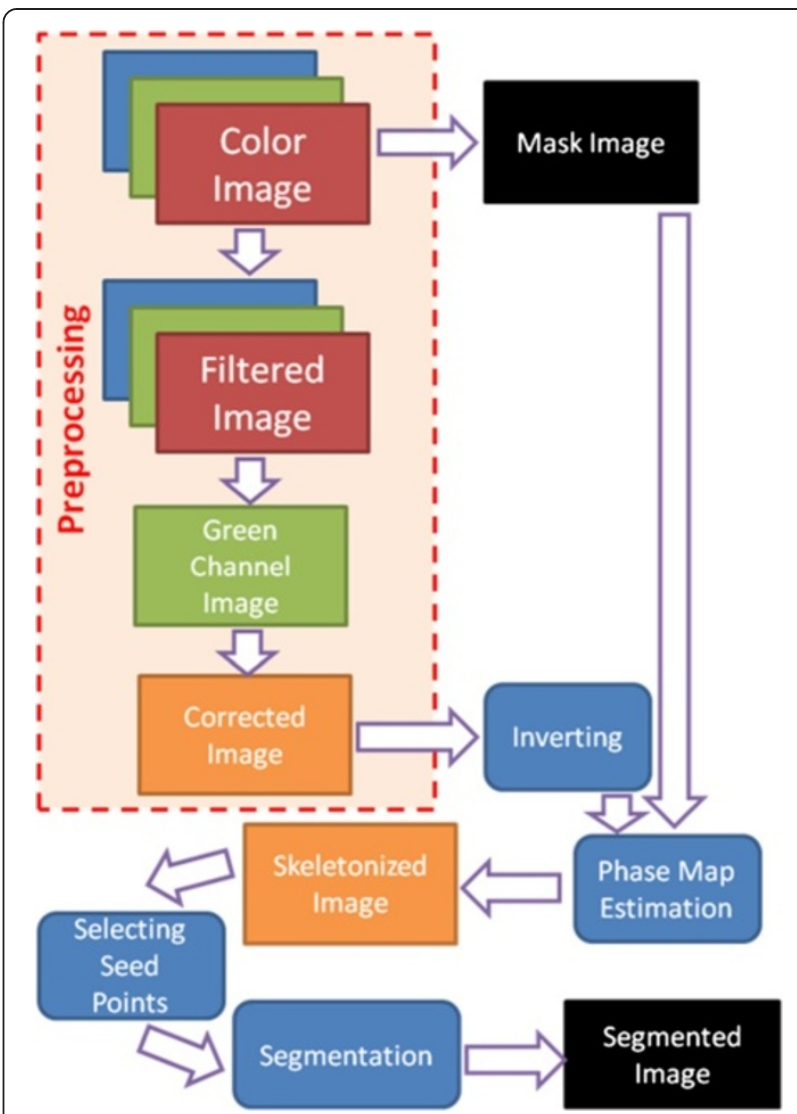

Figure 4 Flowchart of the proposed segmentation algorithm.

one of the following is executed to produce the corrected image:

1. If the input image does not have intensity inhomogeneity, only the histogram-equalized green channel image in the previous step is taken into account as a corrected image.

2. Otherwise, the corrected image is produced by division of those generated images.

To apply the trace-based method on color images, the local geometry for the color image I is obtained by computing the field $\mathbf{K}$ of geometry tensors. $\mathbf{K}$ is the gradient of $\mathbf{I}, \mathbf{K}=\sum{ }_{i=1}^{3} \nabla I_{i} \nabla I_{i}{ }^{T}$, where $\nabla I_{i}=\left[\begin{array}{ll}\partial I_{i} / \partial x, & \partial I_{i} / \partial y\end{array}\right]^{T}$. Moreover, $\mathbf{K}$ is expressed as the following for $\mathbf{I}$ in $R G B$ color space [39]:

$$
\begin{aligned}
& \mathbf{K}=\left[\begin{array}{ll}
\mathrm{k}_{11} & \mathrm{k}_{12} \\
\mathrm{k}_{21} & \mathrm{k}_{22}
\end{array}\right]=\left[\begin{array}{cc}
R_{x}^{2}+G_{x}^{2}+B_{x}^{2} & R_{x} R_{y}+G_{x} G_{y}+B_{x} B_{y} \\
R_{y} R_{x}+G_{y} G_{x}+B_{y} B_{x} & R_{y}^{2}+G_{y}^{2}+B_{y}^{2}
\end{array}\right], \text { where } \\
& R_{x}=\partial I_{1} / \partial x, G_{x}=\partial I_{2} / \partial x \text { and } B_{x}=\partial I_{3} / \partial x \\
& R_{y}=\partial I_{1} / \partial y, G_{y}=\partial I_{2} / \partial y \text { and } B_{y}=\partial I_{3} / \partial y
\end{aligned}
$$




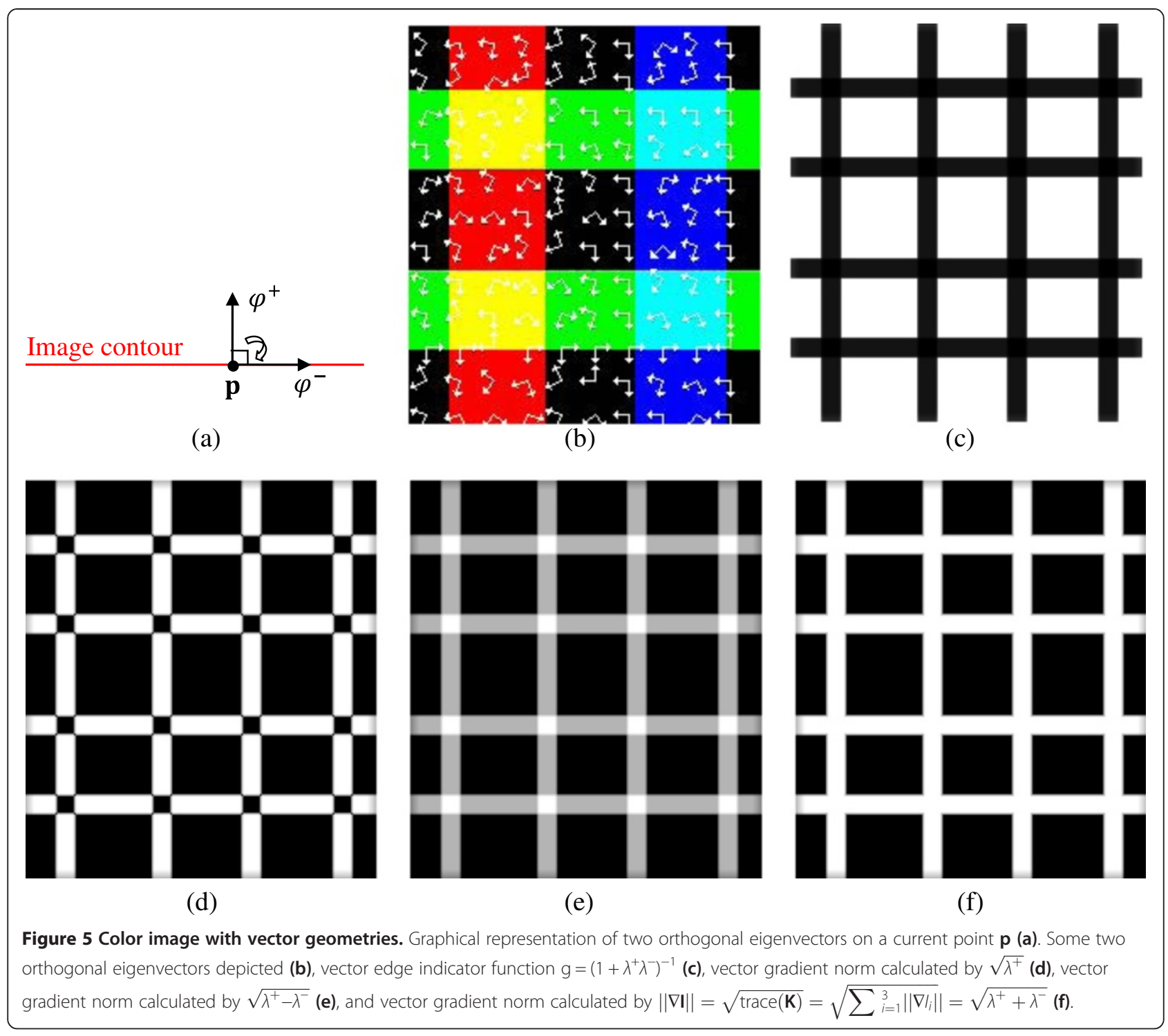

The positive eigenvalues $\lambda^{ \pm}$and the orthogonal eigenvectors $\varphi^{ \pm}$of $\mathbf{K}$ are calculated as

$$
\begin{aligned}
& \lambda^{ \pm}=\left(\mathrm{k}_{11}+\mathrm{k}_{22} \pm \sqrt{\left(\mathrm{k}_{11}-\mathrm{k}_{22}\right)^{2}+4 \mathrm{k}_{12}^{2}}\right) / 2 \text {, and }
\end{aligned}
$$

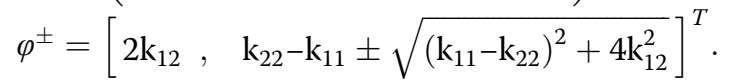

$\mathbf{K}_{\sigma}=\mathbf{K} * G_{\sigma}$ is obtained by eliminating noise via the Gaussian filter $G_{\sigma}$, and a more stable geometry is generated. Here, " is the convolution operator. $\mathbf{K}_{\sigma}$ is a good predictor of the local geometry of $\mathbf{I}$. The spectral elements of $\mathbf{K}_{\sigma}$ give the color-valued variations such as edge strength by means of the eigenvalues $\lambda^{ \pm}$, and they also give the

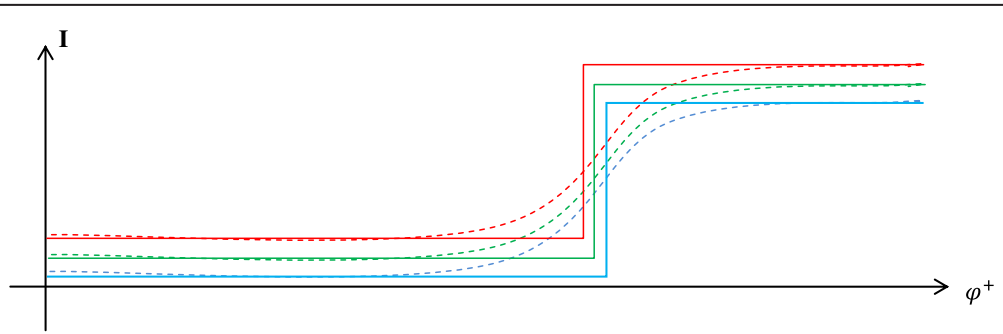

Figure 6 Vector edge enhancement (solid lines) based on vector shock filter. Each image channel smoothed (dashed lines) is sharpened without blurring artifact. 


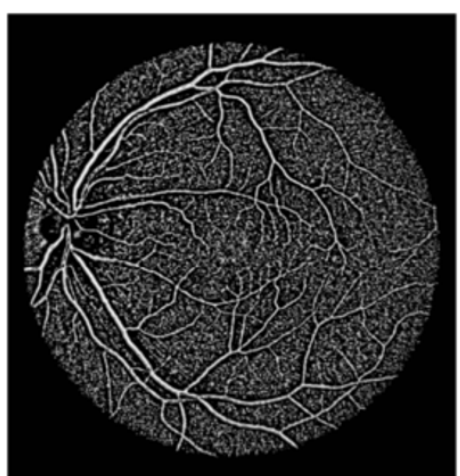

(a)

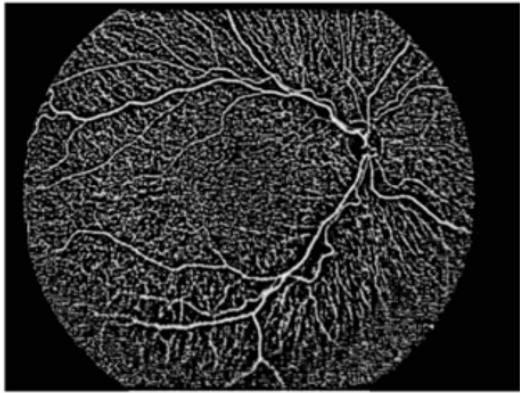

(b)

Figure 7 Image features of the green channel components for retinal fundus images in Figure 1. Edges from phase map [24] (a, b). Note that extremely slim vessel could not be smoothly estimated.

corners and edge directions of the local image structures by means of the eigenvectors $\varphi^{-} \perp \varphi^{+}$(Figure 5). More clearly, eigenvalues $\lambda^{ \pm}$give some information about the active point as follows:

1. If $\lambda^{+} \cong \lambda^{-} \cong 0$, then the point may be in a homogenous region.
2. If $\lambda^{+} \gg \lambda^{-}$, then the point may be on an edge.

3. If $\lambda^{+} \cong \lambda^{-} \gg 0$, then the point may be on a corner.

Tschumperlé et al. [39] suggested designing a particular field $\mathbf{T}: \Omega \rightarrow \mathrm{P}(2)$ of diffusion tensors to define the specification of the local smoothing method for the regularization process. It should be noticed that $\mathbf{T}$,

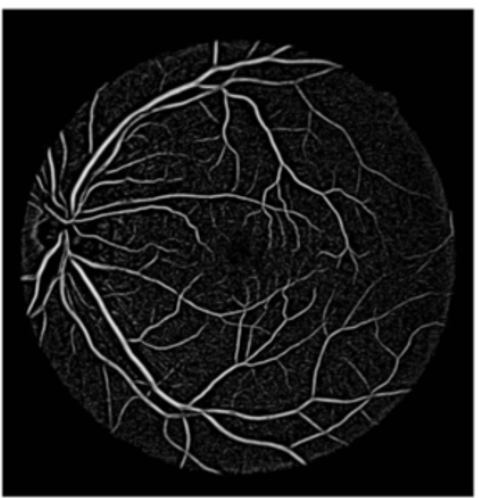

(a)

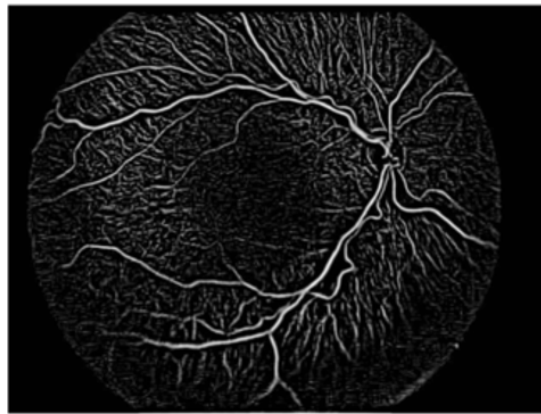

(d)

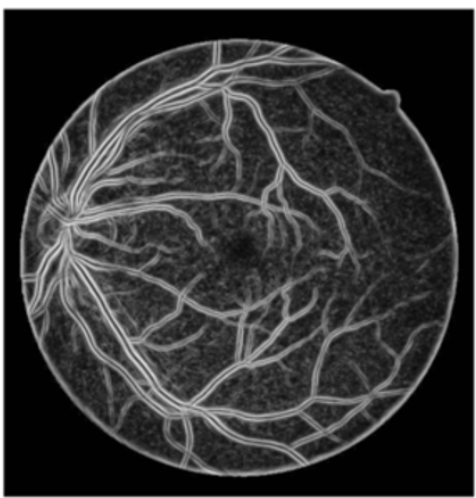

(b)

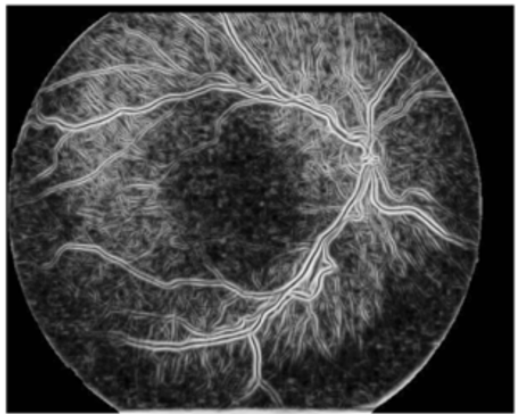

(e)

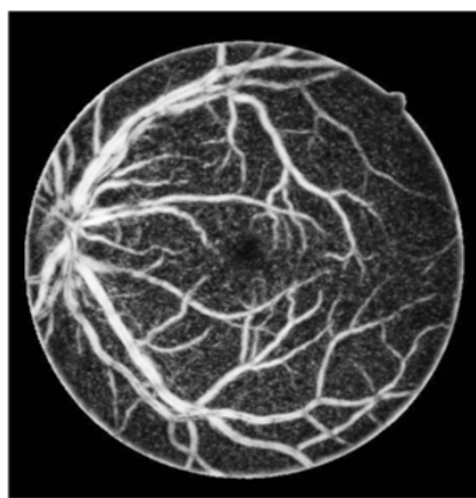

(c)

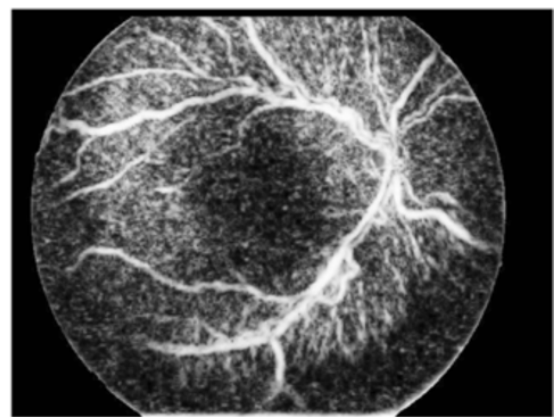

(f)

Figure 8 Features of the green channel components for retinal fundus images in Figure 1. Image edges are shown in (a) and (d), lines are shown in (b) and (e), and structures from our modified phase map approach are shown in (c) and (f). Note that our preprocessing step is not applied on these test images. 
depended on the local geometry of $\mathbf{I}$, can be defined in terms of the spectral elements $\lambda^{ \pm}$and $\varphi^{ \pm}$of $\mathbf{K}_{\sigma}$.

$$
\mathbf{T}=\mathrm{s}^{-}\left(\lambda^{+}, \lambda^{-}\right) \varphi^{-} \varphi^{-T}+\mathrm{s}^{+}\left(\lambda^{+}, \lambda^{-}\right) \varphi^{+} \varphi^{+T}
$$

Here, $s^{ \pm}: \mathbb{R}^{2} \rightarrow \mathbb{R}$ are smoothing functions (along $\varphi^{ \pm}$), and they change depending on the type of application. Sample functions for image smoothing are proposed in [39] as $s^{-}\left(\lambda^{+}, \lambda^{-}\right)=\left(1+\lambda^{+}+\lambda^{-}\right)^{-a_{1}}$ and $s^{+}\left(\lambda^{+}, \lambda^{-}\right)=$

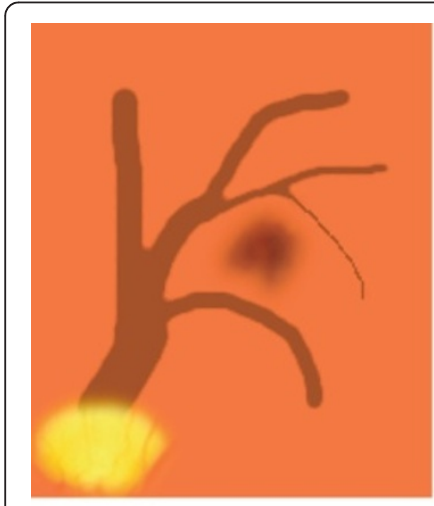

(a)

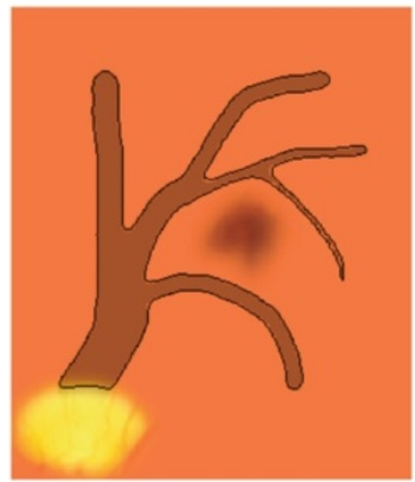

(e)

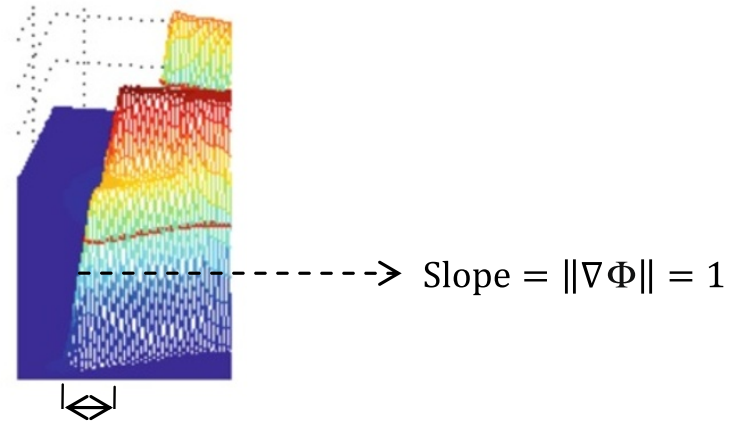

Band region $=2 c_{0}=10$

(i)

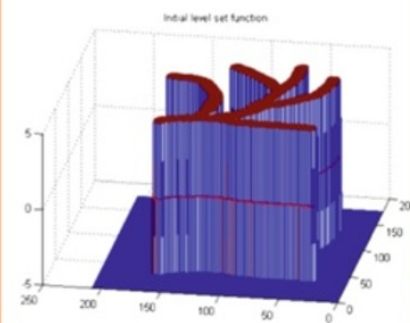

(b)

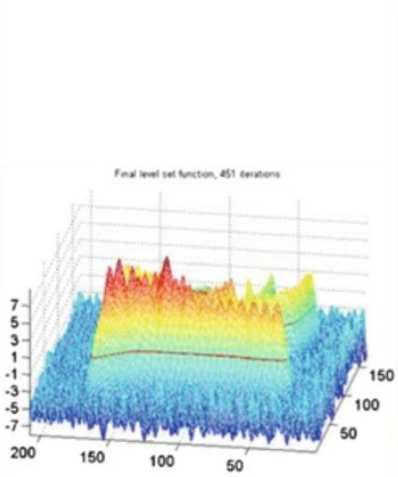

(f)

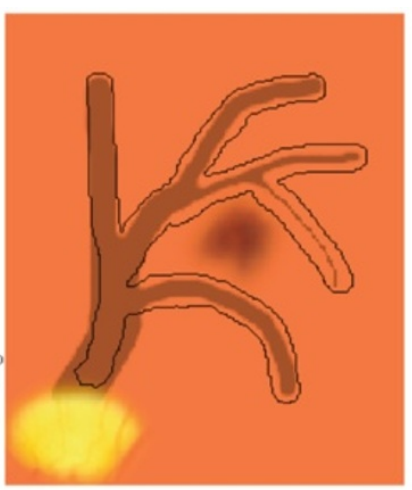

(c)

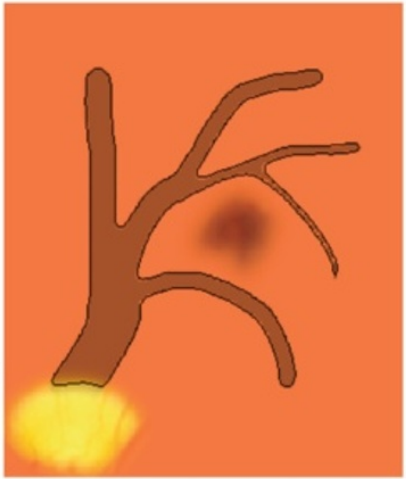

(g)

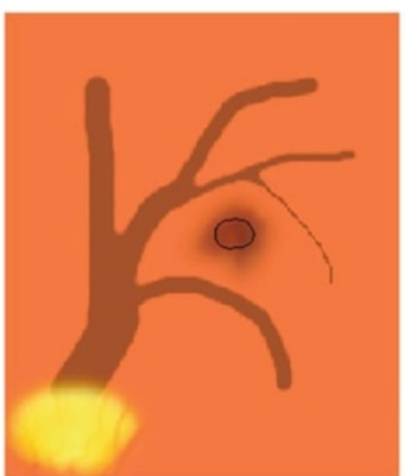

(j)

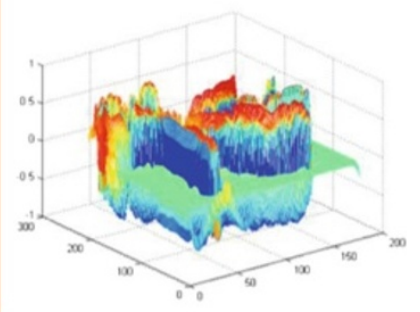

(d)

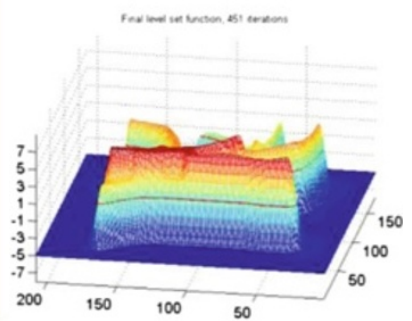

(h)

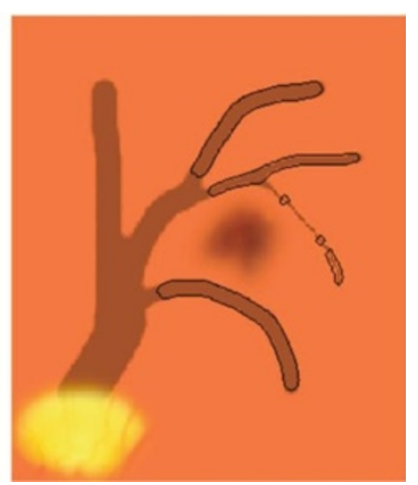

(k)

Figure 9 Level set evolution. Synthetic image (a), initialization of the level set function with a binary function using $c_{0}=5$ (b), and its initial zerolevel contour (c), image edges from modified phase map (d), final level set contours based on the proposed method using the potential function $P_{1}$ (e), and its level set function (f), final level set contours based on the proposed method using the potential function $P_{2}$ ( $\mathbf{g}$ ), and its level set function after 451 iterations (h), slope of final level set function in a band region with size of $2 c_{0}$ (i), final zero-level contours of the given image based on DRLSE [25] using the potential function $P_{2}$ and set by a negative-valued $a(\mathbf{j})$ and a positive-valued $a(\mathbf{k})$. In (g), with area functional $A($.$) , the initial contour is$ shrunk and expanded automatically to match the boundary of vessels. With the length functional $L($.), this fitting has become smooth. The initial level set function in (b) is regularized using the regularization functional $R($.$) , and the final, regularized level set function in (h) is obtained.$ 
$\left(1+\lambda^{+}+\lambda^{-}\right)^{-a_{2}}$, where $a_{1}<a_{2}$. The goals of smoothing operation are

1. To process pixels on image edges along the $\varphi^{-}$ direction (anisotropic smoothing)

2. To process pixels on homogeneous regions on all possible directions (isotropic smoothing). In this case, $\mathrm{T} \cong$ identity matrix and then the method behaves as a heat equation

The regularization approach presented by Tschumperlé et al. [39] is used to obtain the local smoothing geometry $\mathrm{T}$, based on the trace operator:

$$
\partial \mathbf{I} / \partial t=\partial I_{i} / \partial t=\operatorname{trace}\left(\mathbf{T} \mathbf{H}_{i}\right)
$$

where $\mathbf{H}_{i}$ is the Hessian matrix of $I_{i}: \mathbf{H}_{i}=$ $\left[\begin{array}{ll}\partial^{2} I_{i} / \partial x^{2} & \partial^{2} I_{i} / \partial x \partial y\end{array}\right]$ $\left.\partial^{2} I_{i} / \partial y \partial x \quad \partial^{2} I_{i} / \partial y^{2}\right]$

To sharpen the color images, the shock filter is applied on each image channel $I_{i}$ only in one direction $\varphi^{+}$of the vector discontinuities [39]. Moreover, a weighting function is added to enhance color image structure without changing the flat regions. As depicted in Figure 6, such a filter is formulized as follows [39]:

$$
\begin{gathered}
\partial I_{i} / \partial t=\left(\mathbf{s}^{+}\left(\lambda^{+}, \lambda^{-}\right)-1\right) \operatorname{sign}\left(\varphi^{+T} \mathbf{H}_{i} \varphi^{+}\right)\left|I_{i_{\left(\varphi^{+}\right)} \mid}\right|, \text {where } \\
E_{i_{\left(\phi^{+}\right)}=\varphi^{-} E^{-}+\varphi^{+} E^{+},}=\left\{\begin{array}{cc}
0 & \text { if } \frac{\partial I_{i(b)}}{\partial x} \times \frac{\partial I_{i(f)}}{\partial x}<0 \\
\min \left(\frac{\partial I_{i(b)}}{\partial x}, \frac{\partial I_{i(f)}}{\partial x}\right) & \text { else }
\end{array}\right. \\
E^{+}=\left\{\begin{array}{cc}
0 & \text { if } \frac{\partial I_{i(b)}}{\partial y} \times \frac{\partial I_{i(f)}}{\partial y}<0 \\
\min \left(\frac{\partial I_{i(b)}}{\partial y}, \frac{\partial I_{i(f)}}{\partial y}\right) & \text { else }
\end{array}\right.
\end{gathered}
$$

Here, $s^{+}: \mathbb{R}^{2} \rightarrow \mathbb{R}, \mathrm{s}^{+}()=.\left(1+\lambda^{+}+\lambda^{-}\right)^{-0.5}$ is a decreasing function, and sub-indexes $b$ and $f$ stand for backward and forward finite differences, respectively.

The methods based on color information are compatible with all local geometric properties expressed above: $\mathbf{I}_{(t+1)}=\mathbf{I}_{(t)}+\tau_{1} \partial \mathbf{I}_{(t)} / \partial t$, where $\tau_{1}$ is an adapting time step. The adapting time step $\tau_{1}$ is set by the following inequality: $\tau_{1} \leq 20 / \max \left(\max _{\mathbf{p}}\left(\partial \mathbf{I}_{(t)}(\mathbf{p}) / \partial t\right), \min _{\mathbf{p}}\right.$ $\left.\left(\partial \mathbf{I}_{(t)}(\mathbf{p}) / \partial t\right)\right)$.

\subsection{Modified phase map estimation}

Another important step followed in preprocessing retinal fundus images is developing an efficient method for estimation of the image structures in cases, for instance, where retinal vessel network contains slim and lengthy vessels with weak edge intensities. According to our experiment, edge-based level set image segmentation methods give the best results on images that have only structure information in the segmented regions. Although the method [25] described above could segment objects in MRIs and other common medical image formats with reasonable success, it may fail to segment retinal vasculature successfully, due to vessels with weak edge properties. Therefore, an alternative image structure based on the phase map of the image is employed. It should be noted that neither the phase congruencybased method [40] nor the phase map-based approach [24] (see Figure 7) generates adequate structure information for segmentation of vasculature in fundus images [30]. Therefore, we combine these two methods as described below to improve the phase map.

The log-Gabor filter can efficiently extract image features such as edges and corners without missing any weak object boundaries. This filter, generated in frequency domain, is a version of logarithmic transformation of the

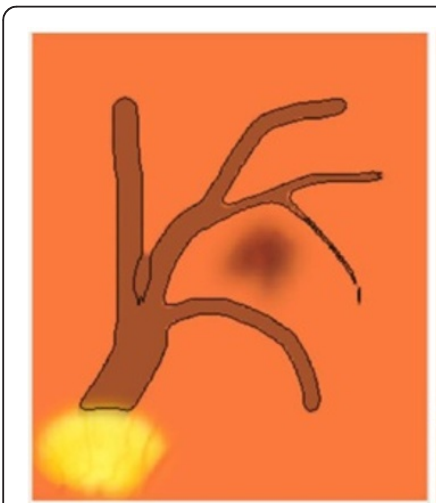

(a)

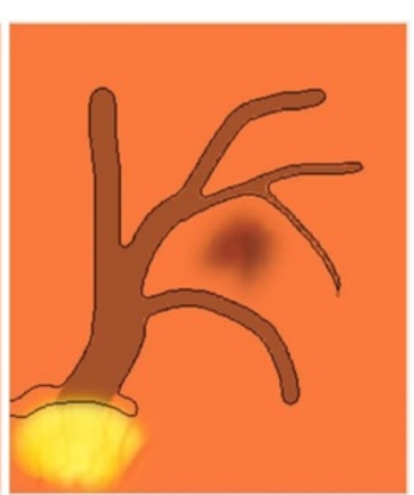

(b)

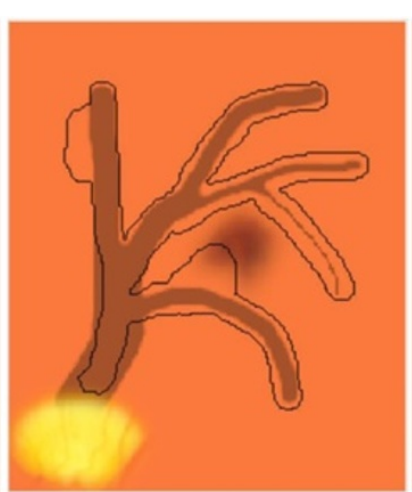

(c)

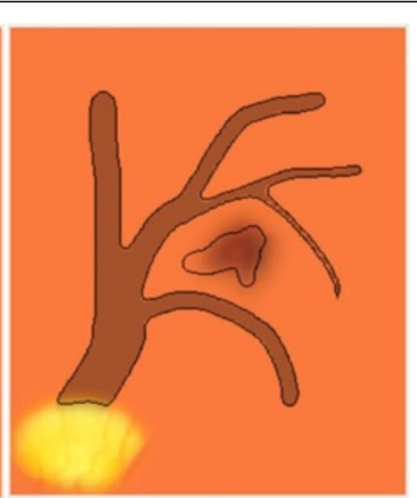

(d)

Figure 10 Failure of the proposed method. The level set function is initialized using the function in Figure 8 for the first two images. Final level set contours based on the proposed method using heat equation as a potential function (a), final level set contours based on the proposed method using the potential function $P_{2}$ without preprocessing (b), binary mask for initialization of the level set function (c), and final level set contours based on the proposed method using the potential function $P_{2}$ (d). 
Table 1 Formulas of some variational image segmentation methods

\begin{tabular}{lll}
\hline Group & Method & Formula \\
\hline Edge-based & GAC [23] & $\vartheta\|\nabla \Phi\| \operatorname{div}\left(g \frac{\nabla \Phi}{\|\nabla \Phi\|}\right)+a g\|\nabla \Phi\|$ \\
& PBLS [24] & $\vartheta\|\nabla \Phi\| \operatorname{div}\left(\frac{\nabla \Phi}{\|\nabla \Phi\|}\right)-a\|\nabla \Phi\| \Re\left(\hat{q}_{\text {PBLS }}\right)$ \\
& DRLSE [25] & $\mu \operatorname{div}(D(\|\nabla \Phi\|) \nabla \Phi)+\vartheta \delta_{\varepsilon}(\Phi) \operatorname{div}\left(g \frac{\nabla \Phi}{\|\nabla \Phi\|}\right)+a g \delta_{\varepsilon}(\Phi)$ \\
Region-based & ARLS [28] & $\mu\left(\nabla^{2} \Phi-\operatorname{div}\left(\frac{\nabla \Phi}{\|\nabla \Phi\|}\right)\right)+\vartheta \delta_{\varepsilon}(\Phi) \operatorname{div}\left(\|\nabla \Phi\|^{s\left(\nabla\left(* G_{\sigma}\right)\right)-2} \nabla \Phi\right)+a \delta_{\varepsilon}(\Phi) \nabla^{2}\left(I * G_{\sigma}\right)$ \\
\hline
\end{tabular}

where $e_{i}$ is the data fitting function of/in region $\Omega_{i}$. See [33] for more details.

Gabor filter [4], and it has no DC component. In polar coordinates, the filter consists of two components, the radial part and the angular part. These two components are combined to create the log-Gabor filter, which is the transfer function formulated as follows [40]:

$$
G_{l}(r, \theta)=\exp \left(-\frac{\left(\log \left(r / f_{0}\right)\right)^{2}}{2 \sigma_{r}^{2}}-\frac{\left(\theta-\theta_{0}\right)^{2}}{2 \sigma_{\theta}^{2}}\right)
$$

Here, $(r, \theta)$ stands for the polar coordinates, $f_{0}$ is the center frequency, $\theta_{0}$ is the orientation angle (direction), $\sigma_{r}=\log \left(v / f_{0}\right)$ defines the scale bandwidth, and $\sigma_{\theta}$ defines the angular bandwidth. In order to keep the shape ratio of the filter constant, the term $v / f_{0}$ must also be kept constant for varying $f_{0}[40]$.

The log-Gabor filter can be efficiently used to generate the phase map instead of the gradient norm in image segmentation $[24,40]$. The image is filtered at different scales in at least three uniformly distributed directions to grab the poor contrast and vasculature with varying width [24]. The filter output is complex in the time domain, where real and imaginary parts consist of line and edge information, respectively. Filter responses in each scale for all directions must be combined to obtain a rotationally invariant phase map. The absolute value of the imaginary parts is taken to avoid an elimination [24].
With these in mind, the modified phase map $q$ is obtained as in Equation 4:

$$
q=\frac{\sum_{k=1}^{O} \sum_{l=1}^{S}\left\|\bar{q}_{k, l}\right\|^{\beta} \bar{q}_{k, l}}{\sum_{k=1}^{O} \sum_{l=1}^{S}\left\|\bar{q}_{k, l}\right\|^{\beta}}
$$

Here, $\bar{q}_{k, l}=\mathfrak{R}\left(q_{k, l}\right)+\left|\mathfrak{I}\left(q_{k, l}\right)\right| \sqrt{-1}, O$ is the number of the orientation angles, $S$ is the number of the scales, $\bar{q}_{k, l}$ is the filter response based on the corrected phase, and $\beta$ is a weighting parameter. The normalization $\hat{q}=q\|q\| /$ $\left(\| q^{2}+\sigma_{q}^{2}\right)$ is also used to regularize the phase map. Here, $\sigma_{q}$ stands for a threshold used to reduce noise effect [24]. Since edges align with the zero crossings of the real part of the phase map, the function $\Re(\hat{q})$ can be used to estimate image edges as in [24]. Moreover, $\mathfrak{J}(\hat{q})$ gives image lines, and the norm of the filter response, formulated as $\|\hat{q}\|=$ $\sqrt{\Re(\hat{q})^{2}+\mathfrak{I}(\hat{q})^{2}}$, gives the strength of the image structure. So, the image structures of the green channel of retinal fundus images are estimated efficiently and correctly by using the log-Gabor filter as seen in Figure 8.

\subsection{Structure-based level set segmentation method}

A novel structure-based variational method is proposed in this study in order to trace retinal vasculature. The level set function in [25] can be discretized more easily compared to

\begin{tabular}{|c|c|}
\hline Method & Parameters \\
\hline $\begin{array}{l}\text { Proposed preprocessing } \\
\text { Log-Gabor filter [40] and modified phase map } \\
\text { GAC [23] }\end{array}$ & $\begin{array}{l}\text { Amplitude of the trace-based filter is } 30 \text {, amplitude of the shock filter is } 45 \text {, } \\
\tau_{1}=\text { updated at each iteration, other parameters of these filters are kept as } \\
\text { in the related study [39], and kernel size of median filter is } 25 \times 25 \text {. } \\
f_{0}(x)=\left(3 \times 2.1^{(x-1)}\right)^{-1}, 1 \leq x \leq S=3, \sigma_{r}=\log (0.55), \\
\sigma_{\theta}=1.2, \theta_{0}(x), 1 \leq x \leq 0=8, \beta=1, \text { and } \sigma_{q}=3 . \\
\tau_{2}=0.2, \vartheta=1 \text {, and } a=0.3\end{array}$ \\
\hline PBLS [24] & $\tau_{2}=$ updated at each iteration, $\vartheta=0.07$, and $a=1$ \\
\hline DRLSE [25] & $\tau_{2}=5, \sigma=1.5, \mu=0.04, \vartheta=5, a= \pm 1.5$, and $c_{0}=2$ \\
\hline ARLS [28] & $\tau_{2}=5, \sigma=1.4, \mu=0.04, \vartheta=2.7, a= \pm 1$, and $c_{0}= \pm 1$ \\
\hline Proposed segmentation & $\tau_{2}=1, \mu=0.2, \vartheta=0.6, a=3$, and $c_{0}=\{2,5\}$ \\
\hline RBLSE [33] & $\tau_{2}=0.1, \sigma=4, \mu=1, \vartheta=0.01 \times 255^{2}$, and $c_{0}=1$ \\
\hline
\end{tabular}

Table 2 Parameter values of the methods 
other methods in the literature since it has a level set regularization term. The discretization process uses center/forward difference model instead of other complex discretization schemes [23,24]. For instance, in the GAC model in [23], the upwind method is used for the calculation of the gradient norm of the level set function $\Phi$, and for the re-initialization of the level set function $\Phi$, essentially non-oscillatory (ENO) scheme is employed. Therefore, the same level set function regularization term of the DRLSE method [25] is used in the proposed method.

In the DRLSE method [25], the formulas of $R(\Phi)=\int_{\Omega}$ $P(\Phi) d \mathbf{p}, \quad L(\Phi)=\int_{\Omega} \quad g \delta_{\varepsilon}(\Phi)\|\nabla \Phi\| d \mathbf{p} \quad$ and $\quad A(\Phi)=\int_{\Omega}$ $g H_{\varepsilon}(-\Phi) d \mathbf{p}$ are employed for segmentation. Here, $P($.$) is$ a potential function. The length functional $L($.) smoothes the zero-level contour. The area functional $A($.$) helps ac-$ celerate the level set evolution when the initial contour is located far away from the object boundaries. For demonstration, see Figure 9.

In edge-based level set approaches, a smooth edge indicator function is generally obtained from the gradient norm of the Gaussian-filtered image. One choice is $g=\left(1+\left\|\nabla\left(G_{\sigma} * I\right)\right\|^{2}\right)^{-1}$. The edge indicator function $g$ carries key information to locate the zero-level contour. $H_{\varepsilon}$ and $\delta_{\varepsilon}=H_{\varepsilon}^{\prime}$ are finite-width approximations of the Heaviside function and Dirac-delta for $\varepsilon$ :

$$
\begin{array}{r}
H_{\varepsilon}(x)=\left\{\begin{array}{lc}
\frac{1}{2}\left[1+\frac{\pi}{\varepsilon}+\frac{1}{\pi} \sin \left(\frac{\pi x}{\varepsilon}\right)\right],|x| \leq \varepsilon \\
1, & x>\varepsilon \\
0, & x<-\varepsilon
\end{array}\right. \text { and } \\
\delta_{\varepsilon}(x)=\left\{\begin{array}{cc}
\frac{1}{2 \varepsilon}\left[1+\cos \left(\frac{\pi x}{\varepsilon}\right)\right], & |x| \leq \varepsilon \\
0, & |x|>\varepsilon
\end{array}\right.
\end{array}
$$

where, in general, the parameter $\varepsilon$ is set to 1.5 .

The level set function regularization term should have a minimum to maintain the signed distance property of $\|\nabla \Phi\|=1$ in a band region around the zero-level contour as depicted in Figure 9i, instead of the heat equation [25] that enforces $\|\nabla \Phi\|=0$, eventually. So, the solution, based on the potential function $P_{1}(|| \nabla \Phi||)=0.5(|| \nabla \Phi||-1)^{2}$, is formulated as follows [25]:

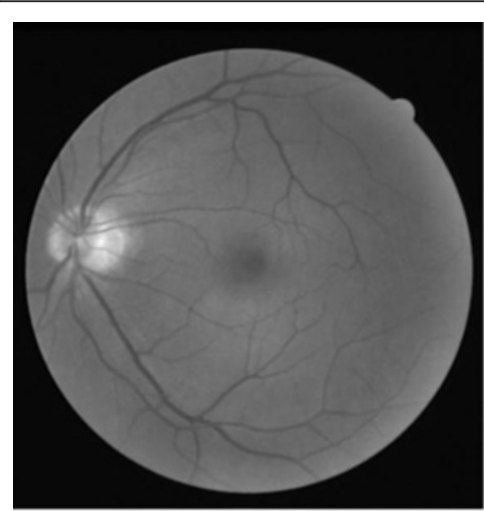

(a)

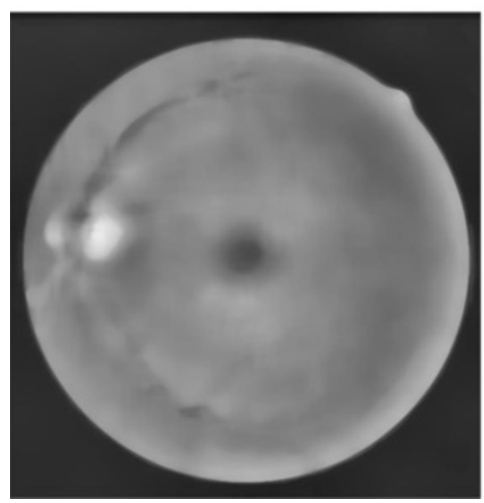

(d)

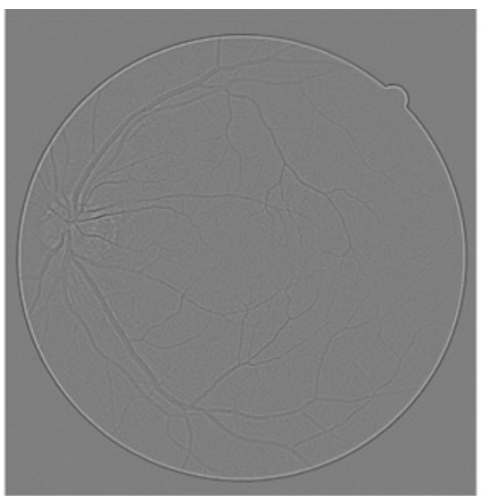

(b)

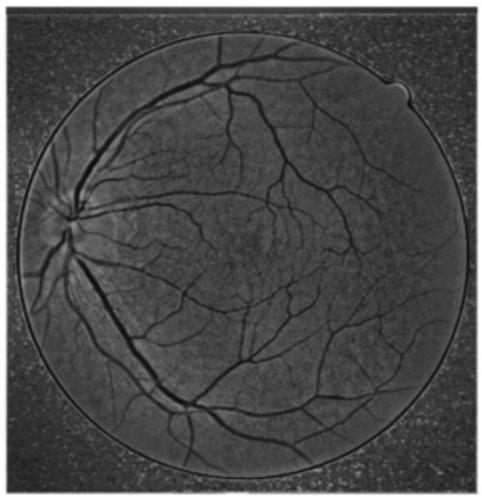

(e)

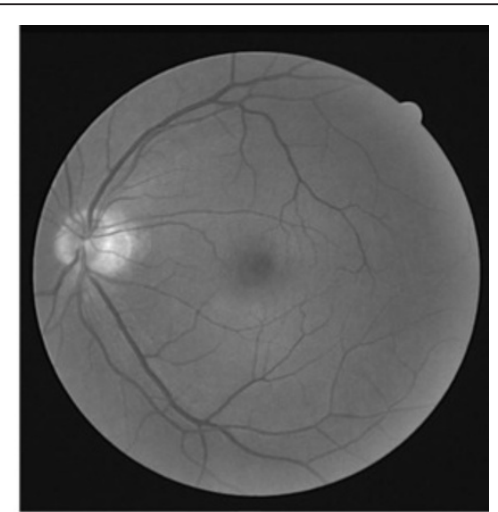

(c)

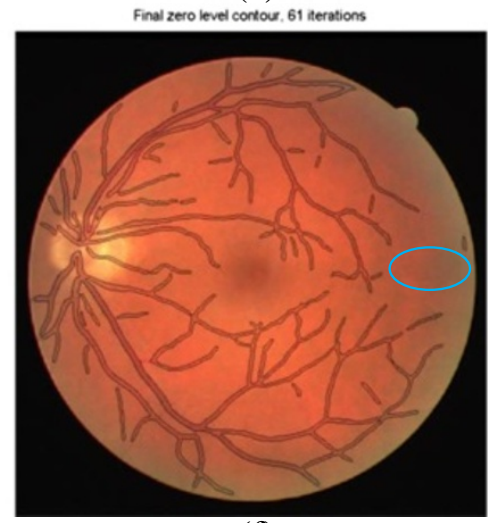

(f)

Figure 11 Preprocessing step and segmentation of non-pathological retinal fundus image obtained from DRIVE dataset. The smoothed image using the scalar approach [19,38] (a), the image generated by subtracting the original green channel image from the smoothed one (b), the sharpened image (c), the estimated intensity inhomogeneity image obtained by using the median filter (d), the corrected image (e), and segmented image using the proposed structure-based level set segmentation method after 61 iterations (f). 


$$
\frac{\partial \Phi_{R}}{\partial t}=\mu \operatorname{div}(D(\|\nabla \Phi\|) \nabla \Phi)=\mu\left(\nabla^{2} \Phi-\operatorname{div}\left(\frac{\nabla \Phi}{\|\nabla \Phi\|}\right)\right)
$$

The sign of $D(\| \nabla \Phi||)=1-(1 / \| \nabla \Phi||)$, where $D(x)=x^{-1}$ $\partial P(x) / \partial x$ indicates the property of the diffusion term based on anisotropic regularization in the following two cases [25]:

1. For $\|\nabla \Phi\|>1$, the diffusion rate $\mu D($.) is positive and the diffusion is forward, which decreases the term $\|\nabla \Phi\|$

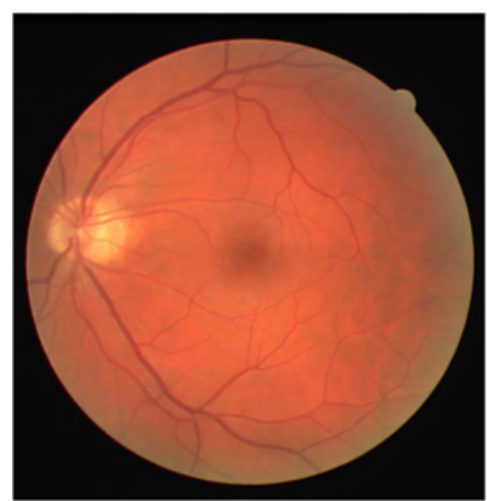

(a)

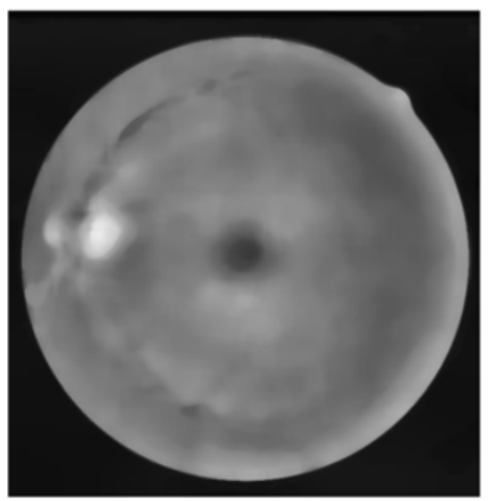

(d)

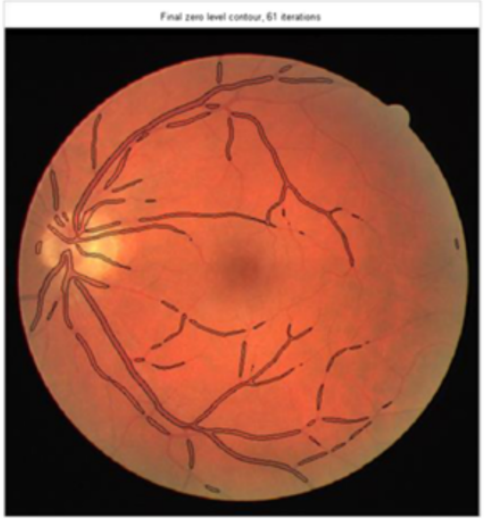

(g)

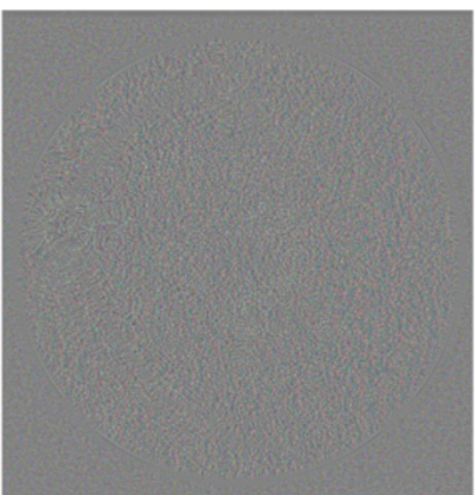

(b)

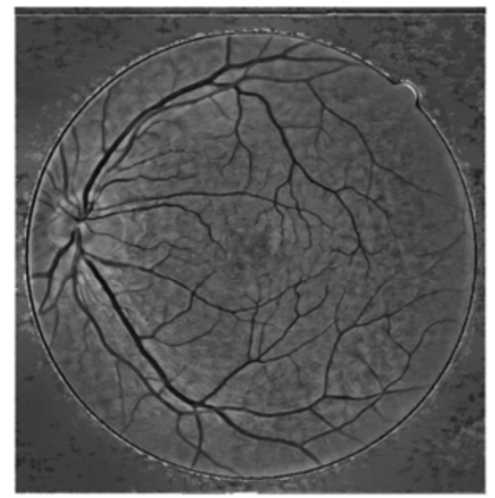

(e)

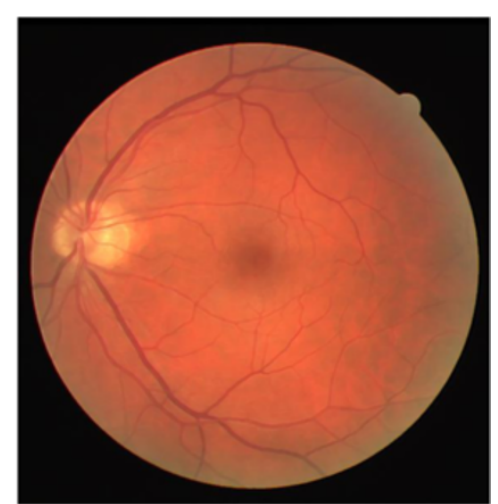

(c)

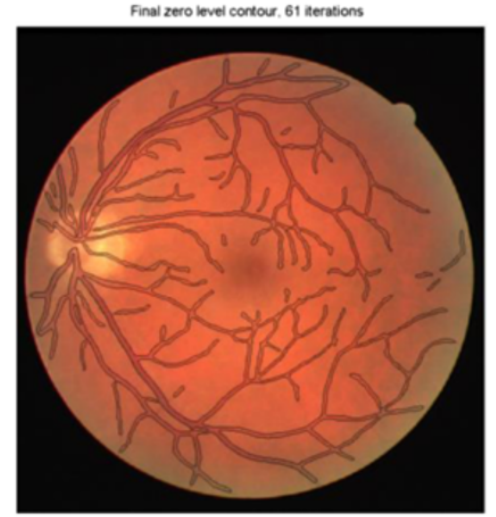

(f)

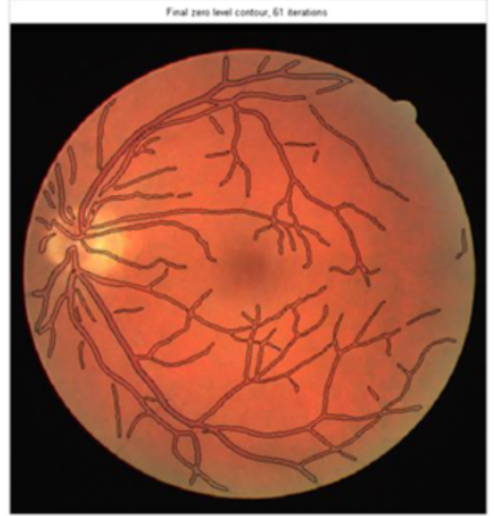

(h)

Figure 12 Preprocessing step and segmentation of non-pathological retinal fundus image obtained from DRIVE dataset. The smoothed image using the trace-based approach [39] (a), the image generated by subtracting the original green channel image from the smoothed one (b), the sharpened image [39] (c), the estimated intensity inhomogeneity image obtained by using the median filter (d), the corrected image based on our approach (e), segmented image using the proposed structure-based level set segmentation method (f), segmented result using only green channel of the given image based on the proposed structure-based level set segmentation method without preprocessing (g), and segmented result using only green channel of the histogram-equalized given image based on the proposed structure-based level set segmentation method without preprocessing after 61 iterations (h). 
2. For $\|\nabla \Phi\|<1$, the diffusion is backward, which increases the term $\|\nabla \Phi\|$

However, this regularization term may cause an unsatisfactory result on the level set function when $\|\nabla \Phi\|$ is close to 0 outside the band region as shown in Figure 9e,f. So, as given in Figure 9g,h, a corrected potential function is given as follows [25]:

$$
P_{2}(x)= \begin{cases}\frac{1}{(2 \pi)^{2}}(1-\cos (2 \pi x)) & \text { if } x \leq 1 \\ \frac{1}{2}(x-1)^{2} & \text { if } x \geq 1 .\end{cases}
$$

In the proposed method, the initial contours have to be set automatically around vessels in order to find the global minimum in a segmented image correctly. Sometimes, there is a risk of getting stuck in a local minimum due to the fact that retinal fundus images include defects such as drusen, GA, etc. So, seed points should be chosen around vessel regions to generate a desirable result. Note that the seed points can be set in or out of vessel areas, but they should be very close to the vessel structures (compare Figures 9 and 10). There is another approach, called the ARLS method [28] in the literature, utilizing automatic initial contours based on Laplacian of Gaussian (LoG) filter. This method is not proper for segmenting retinal vasculature, as the filter is very sensitive to noise, and there is a risk in the automatic initial contours if the retinal fundus image contains pathological regions. On the contrary, in the proposed method, the real part of the modified phase map has zero-crossing boundaries, and the method ensures to find the global minimum if the initial contour is selected around vasculature regions (Figure 9a,b,c,d,e,f,g,h). Therefore, we improve the speed term based on the area functional $A($.$) as$ follows:

$$
\frac{\partial \Phi_{A}}{\partial t}=-\alpha \delta_{\varepsilon}(\Phi) \Re(\hat{\mathrm{q}})
$$

In our method, iso-contours automatically shrink when the contour is outside the object due to the functional of $A($.$) returning a positive contribution, or they$ automatically expand with a negative value in $A($.$) when$ the contour is inside, regardless of the sign of $\alpha$ values as in the existing method [25] (Figure 9j,k).

To eliminate staircasing effect [41] and not to miss weak object boundaries [28], a potential function based on weighted total variation (WTV) model is used as $P_{3}(\Phi)=$ $\frac{\nabla^{s(\nabla(I * G \sigma))}}{s\left(\nabla\left(I * G_{\sigma}\right)\right)}$. Here, $s: \mathbb{R} \rightarrow[1,2)$ is a monotonically decreasing function $[27,28,41]$. Such a function used in the ARSL method [28] is not capable of regularizing zero-level contours because of the smoothed gradient norm which cannot generate image structure. Furthermore, the total variation (TV) model, presented in the PBLS method [24], will not smooth zero-level contours completely, generating unsatisfactory results. Therefore, we suggest a modified oriented Laplacian flow as in Equation 7, originally employed in image denoising [39,42], in order to regularize the zero-level contour:

$$
\frac{\partial \Phi_{L}}{\partial t}=\vartheta \delta_{\varepsilon}(\Phi)\left(\Phi_{\xi \xi}+\mathbf{s}(\|\hat{q}\|) \Phi_{\eta \eta}\right)
$$

where $s(\|\hat{q}\|)=\left(1+\|\hat{q}\|^{2}\right)^{-1}, \Phi_{\zeta \zeta}=\zeta^{T} \mathbf{H} \zeta, \Phi_{\eta \eta}=\eta^{T} \mathbf{H} \eta$, and $\mathbf{H}$ is the Hessian of $\Phi$. The unit vectors $\eta$ and $\zeta$ are represented by the gradient direction and the tangential

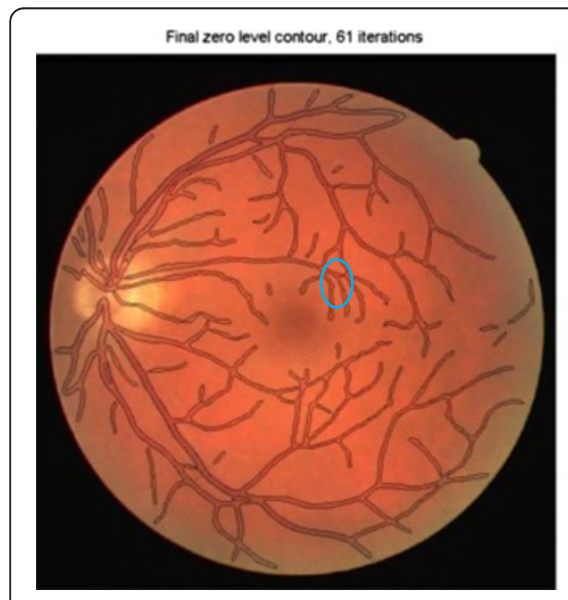

(a)

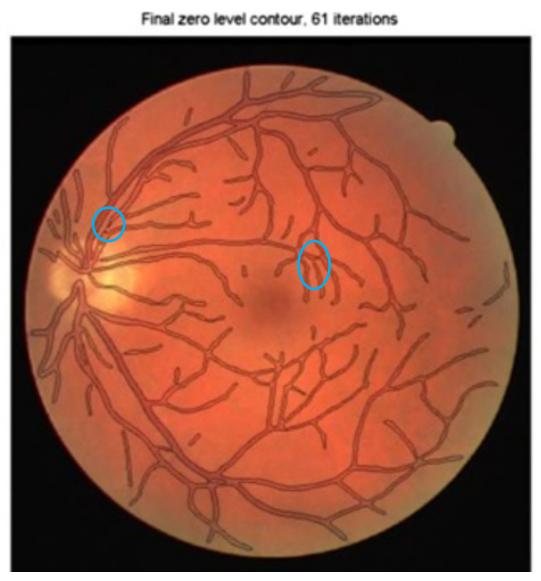

(b)

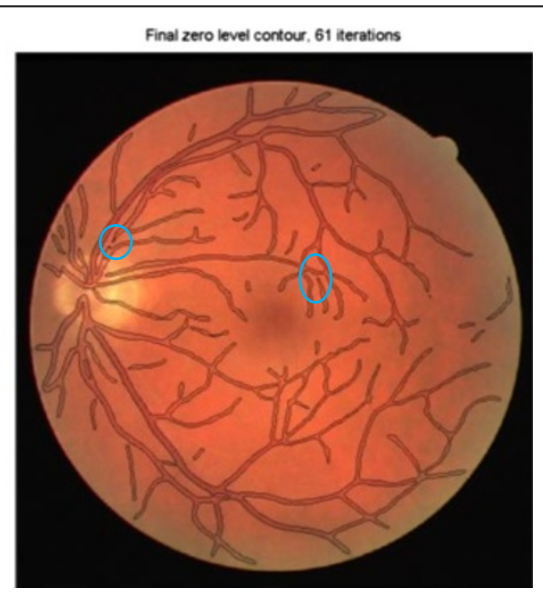

(c)

Figure 13 Level set evolution with setting parameter values for a non-pathological image obtained from DRIVE dataset. $\vartheta=0.4$ and $a=1.5$ (a), $\vartheta=0.8$ and $a=2.5$ (b), and $\vartheta=1$ and $a=3$ (c). 
(its orthogonal) direction, respectively. Here, $\eta=\nabla \Phi /$ $\|\nabla \Phi\|$ and $\zeta=\eta^{\perp}$. s(.) depends on the value of the strength of the image structure $\|\hat{q}\|$, which is generated from phase map. So, along the zero-level contour, the oriented Laplacian flow has a strong smoothing effect. As a result, our approach is more efficient compared to the PBLS method [24] to regularize zero-level contours.

\subsection{Proposed segmentation method}

The proposed method accepts a retinal fundus image in $R G B$ color space as input. Firstly, a simple mask is obtained to exclude the exterior parts of the fundus where the color is in the $0-U$ interval in all three channels (generally very dark regions). Also, an iterated erosion operator whose structure element is $\mathbf{B}=[0,1,0 ; 1,1,1$;

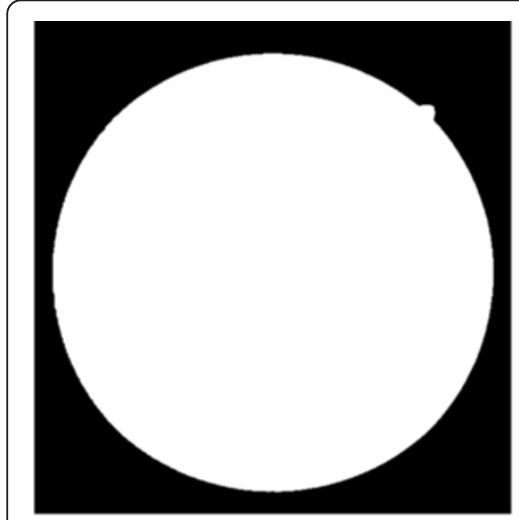

(a)

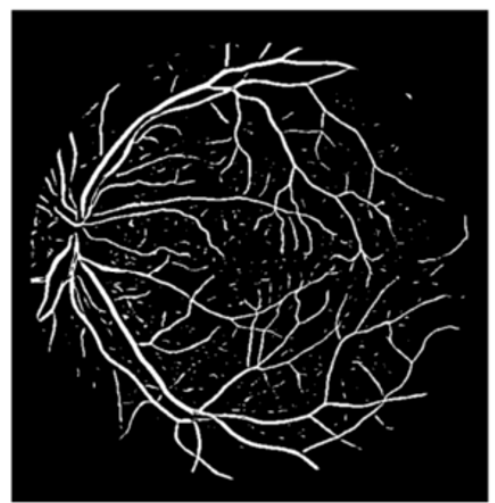

(d)

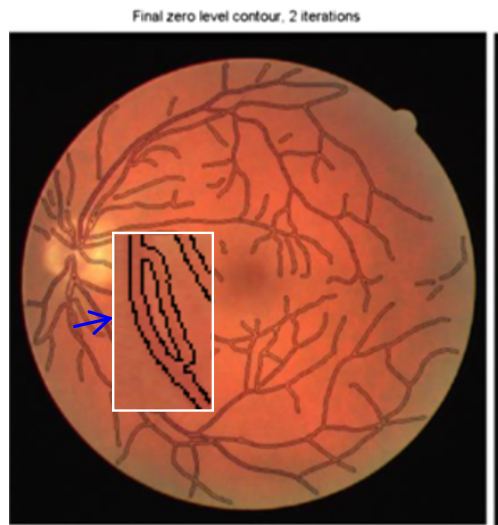

(g)

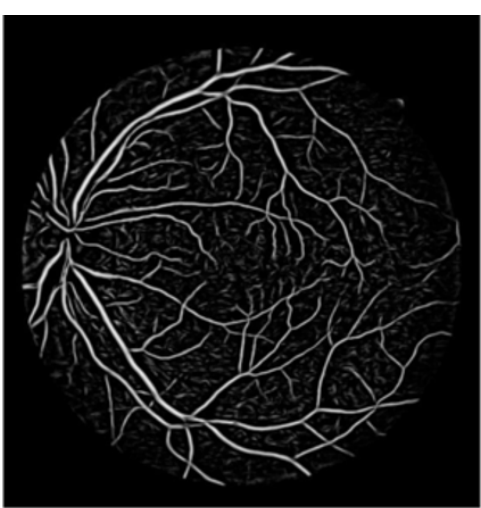

(b)

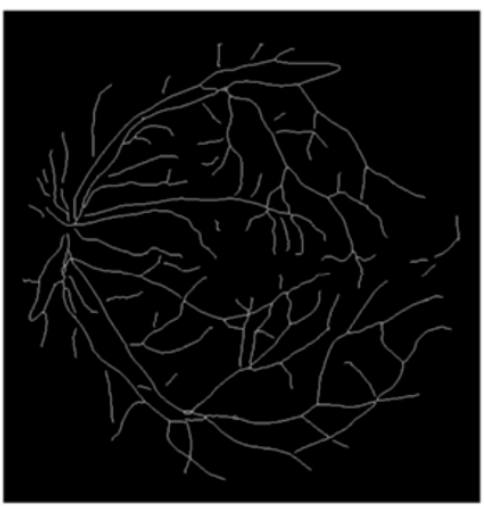

(e)

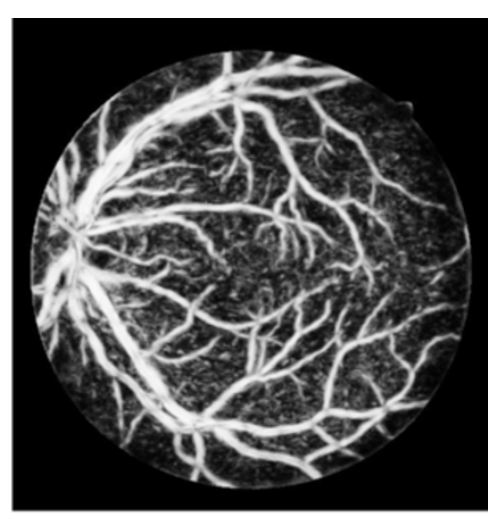

(c)

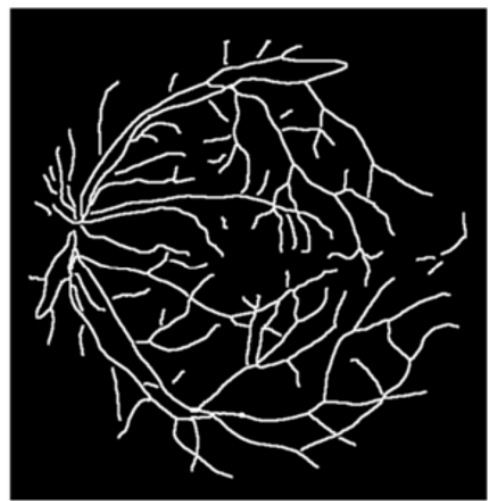

(f)

Final zero level contour, 51 terations

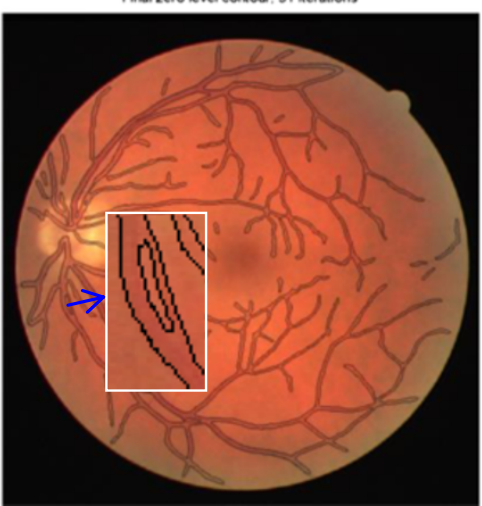

(h)

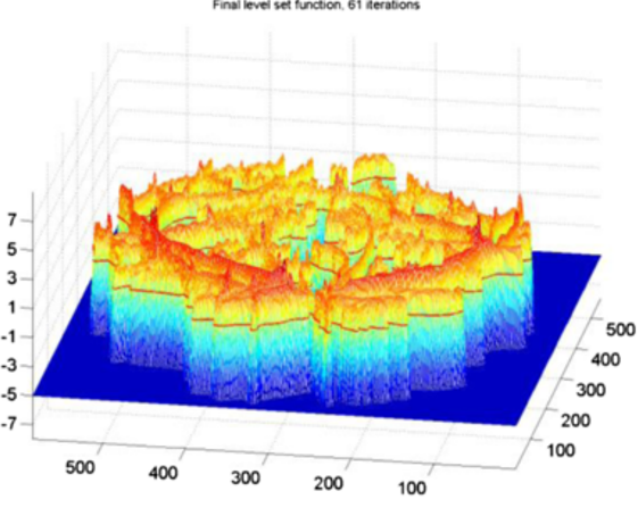

(i)

Figure 14 Segmentation processes of a non-pathological retinal fundus image obtained from DRIVE dataset. Mask image (a), image edge obtained using the proposed phase map (b), image structure obtained using the phase map (c), binary image obtained from (b) using Otsu thresholding [43] (d), skeletonized version of (d) after eliminating outliers (e), dilated version of (e) (f), segmented image using the proposed method after 2 iterations (g), segmented image using the proposed method after 51 iterations (h), and the level set function after 61 iterations (i). 
$0,1,0]^{T}$ is applied on the mask for proper execution. Secondly, a preprocessing step is employed to obtain a corrected image in terms of intensity inhomogeneity. Thirdly, we compute the phase map by using the corrected image as input. Afterwards, to eliminate some small non-blood vessels region, Otsu's method [43] is applied on the processed image. As a result of these processes, a skeleton-based image giving the centerlines of the vasculature is generated with the following steps: (i) remove disconnected pixels, (ii) obtain skeleton-based image, (iii) find junctions, (iv) trace lines (centerlines) and label them, and (v) clean short lines. Here, a threshold value is used to eliminate tiny little short lines called artifacts.

In order to set the optimum initialization of the zerolevel contour, seed points have to be selected around the vasculature according to the centerline obtained based on phase map properties. Here, a morphological dilation operator whose structure element is $\mathbf{B}=[1,1,1 ; 1,1,1$; $1,1,1]^{T}$, is performed on the centerlines to generate a proper initial contour. Finally, the proposed method creates the output by using the structure-based level set method. Our level set function is minimized by using Euler Lagrange and the iterative gradient descent procedure as follows:

$$
\begin{aligned}
\frac{\partial \Phi}{\partial t}= & \mu \operatorname{div}(D(\|\nabla \Phi\|) \nabla \Phi)+\vartheta \delta_{\varepsilon}(\Phi) \\
& \times\left(\Phi_{\zeta \zeta}+\mathbf{s}(\|\hat{q}\|) \Phi_{\eta \eta}\right)-\alpha \delta_{\varepsilon}(\Phi) \Re(\hat{q})
\end{aligned}
$$

Note that values of the edge indicator function $g$, used in $[25]$, are in the $[0,1]$ interval. In the proposed method, the sign of the coefficient $\alpha$ in the level set energy functional can always remain positive in contrast to the earlier method [25] since the function $\mathfrak{R}(\hat{q})$ obtained from the phase map has a different sign around object boundaries.

The proposed level set evolution equation culminates in $\Phi_{(t+1)}=\Phi_{(t)}+\tau_{2} \partial \Phi_{(t)} / \partial t$ where $\tau_{2}$ is a time step, which is set by $\tau_{2} \leq(4 \mu)^{-1}$ based on Courant-Friedrichs-Lewy (CFL) condition with 4-neighbor connectivity [25,44].

The initialization of level set function is important. If the seed points are selected away from the vessel centers and close to pathological regions, the proposed method can fail (wrongly segmenting the pathological region, as well) as shown in Figure 10d.

\section{Experimental results}

The proposed method is tested on DRIVE [3], STARE [11], and our own datasets [2,19] for this study. Our 34 wide-angle fundus images are grabbed from premature infants supplied by the RetCam II camera and delineated by medical experts. The images from different experts are combined to create one ground truth image for each one of the fundus image [1,2]. Some methods used in this study are summarized in Table 1 . The chosen parameters of the algorithm are given in Table 2. Eight uniformly distributed angle directions and three image re-sampling scales for the log-Gabor filter are used in the method. The maximum number of iterations for the

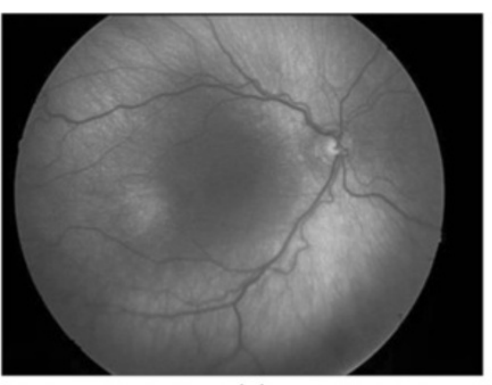

(a)

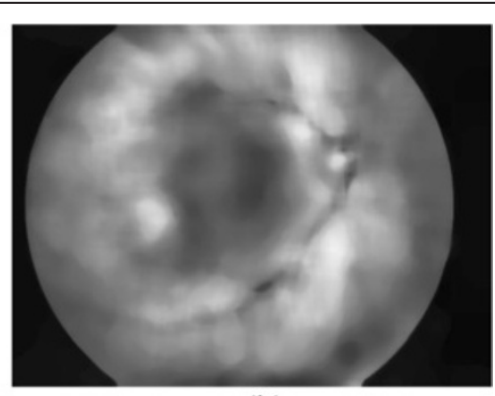

(b)

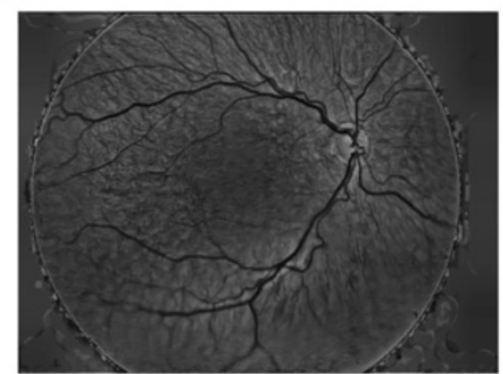

(c)

Figure 15 Preprocessing step and segmentation of a non-pathological retinal fundus image obtained from our dataset. Green channel of the given image in Figure 1a (a), estimated intensity inhomogeneity image that is obtained by using the median filter (b), and the corrected image that is obtained by our method (c). 
main algorithm depends on the radii of the vessel, and for this study, it is experimentally set as $60+1$ (extra regularization of the zero-level contour via level set evolution with $\alpha=0$ ). The threshold values of $U$ for creating mask images are set to 40, 40, and 45, for DRIVE dataset, our dataset, and STARE dataset, respectively. Moreover, small gaps in the created mask image for STARE dataset are filled using a morphological closing operator whose structure element is a disk of radius 10 . In order to eliminate the out of fundus image region, the numbers of iterated erosion operator are set to 8,8 , and 2 for DRIVE dataset, our dataset, and STARE dataset, respectively. The threshold values of short line length are set as 15, 35, and 15 for DRIVE dataset, our dataset, and STARE dataset, respectively. $c_{0}$ values for initializing of level set functions are set to 5, 5, and 2 for DRIVE dataset, our dataset, and STARE dataset, respectively. Here, in all cases except for the 20th image from STARE dataset, second selection is used for preprocessing. First selection is used for preprocessing on 20th image from STARE dataset because this image does not have intensity inhomogeneity. The Neumann boundary condition is employed [25] to solve Equation 8.

The results of the preprocessing step for some test images from DRIVE dataset are seen in Figures 11 and 12. Using the segmented image, on which a scalar approach $[19,38]$ is applied for the preprocessing step, the vasculature cannot be traced truly. This does not happen in our method because we use a trace-based method to smoothen and then a shock filter to sharpen the given image. Both filters work based on the color information unlike the ones in the scalar approach presented in

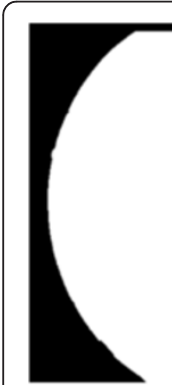

(a)

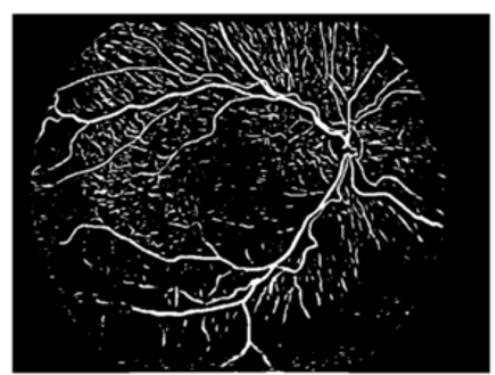

(d)

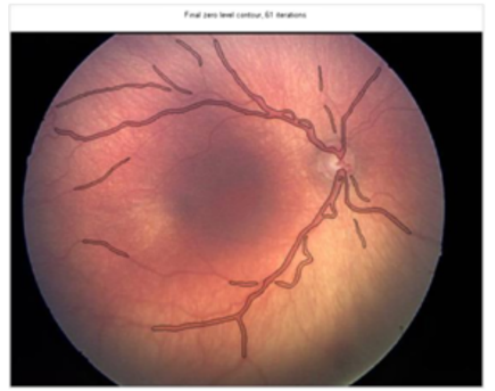

(g)

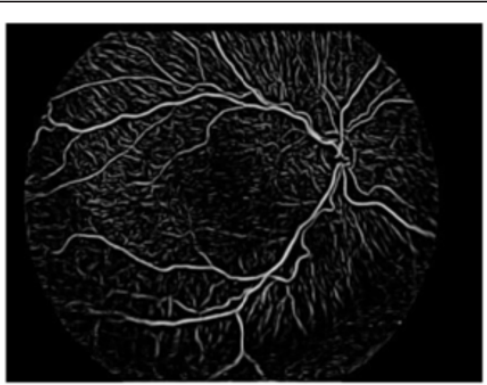

(b)

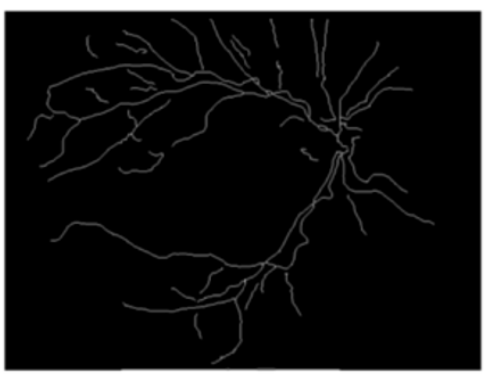

(e)

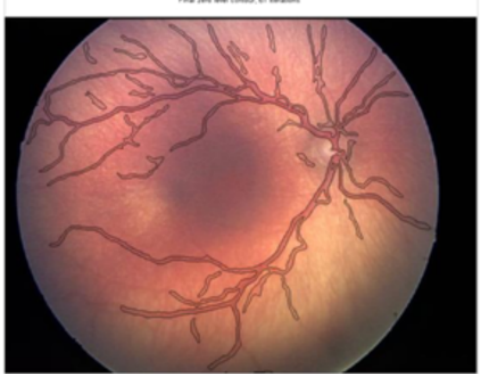

(h)

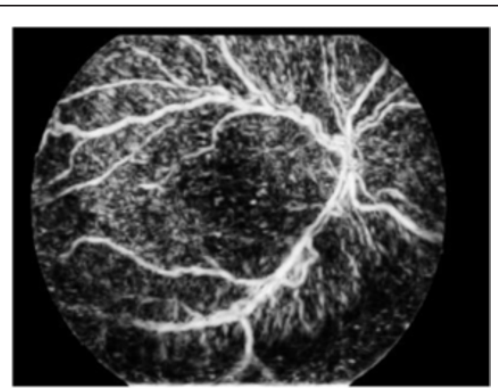

(c)

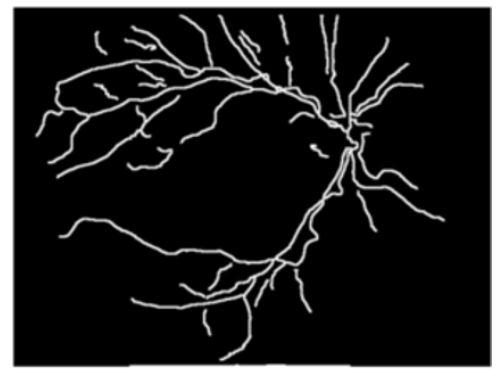

(f)

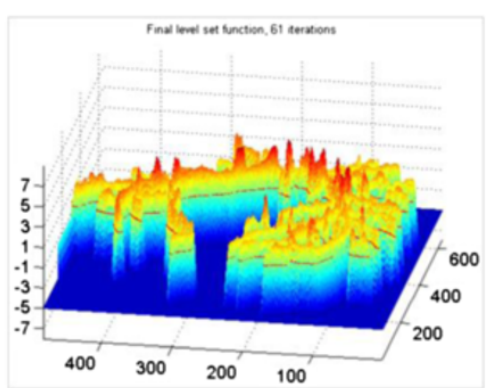

(i)

Figure 16 Segmentation processes of a non-pathological retinal fundus image obtained from our dataset. Mask image (a), image edge obtained using the proposed phase map (b), image structure obtained using the phase map (c), binary image obtained from (b) using Otsu thresholding [43] (d), skeletonized version of (d) after eliminating outliers (e), dilated version of (e) (f), segmented result using only green channel of the given image based on the proposed structure-based level set segmentation method without preprocessing (g), segmented image using the proposed method (h), and the level set function after 61 iterations (i). 
$[19,38]$. Therefore, the image, obtained by our method, is denoised more efficiently and segmented more correctly. While our method produces promising results, we should also indicate that there are still missed retinal vessels. Those missed vessels are very thin with weak edge properties. There are regular retinal vessels with normal dimensions wholly missed with the preprocessing step presented in [19]. Such a region is marked with a blue circle as shown in Figure 11f. In Figure 12b, a difference image between input color image and smoothed version of the input image is shown. The blue channel has noise and seems to contain higher frequencies compared to Figure 11b. Furthermore, images that could not be segmented using the proposed structure-based level set segmentation method without preprocessing are shown in Figure 12g,h.

Figure 13 demonstrates the results of the level set function evolution based on setting the coefficient values $\vartheta$ and $\alpha$ used in the length term regularizing zero-level contour and the speed term accelerating the level set function evolution. $\vartheta$ is set to $0.4,0.8$, and 1 , and $\alpha$ is set to $1.5,2.5$, and 3, respectively, as shown Figure 13a,b,c.

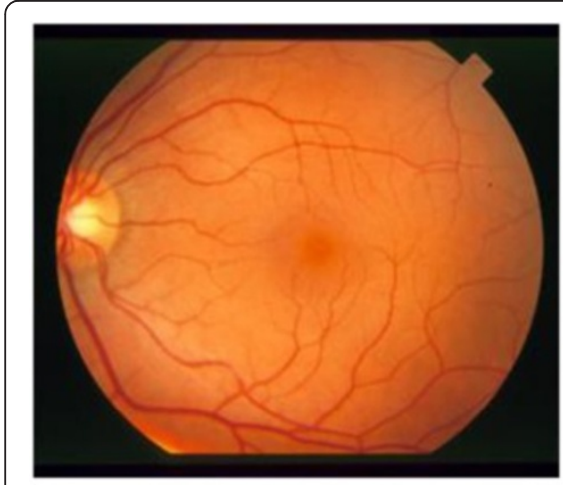

(a)

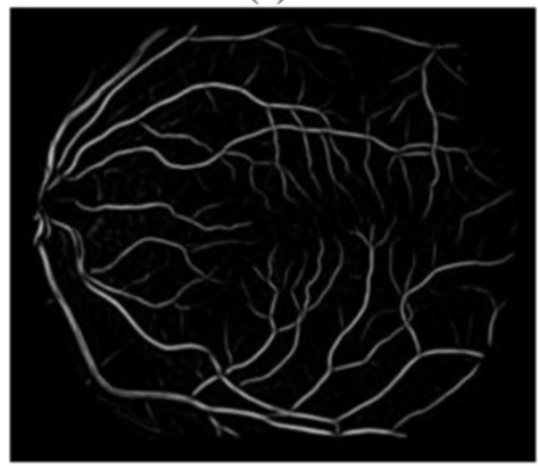

(d)

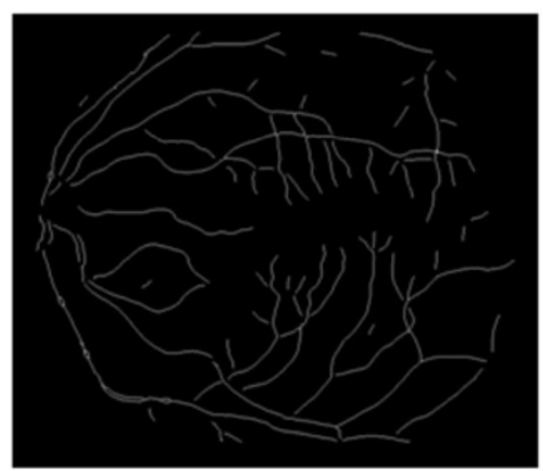

(g)

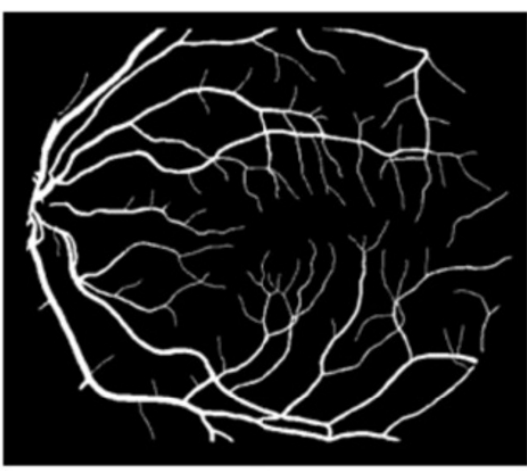

(b)

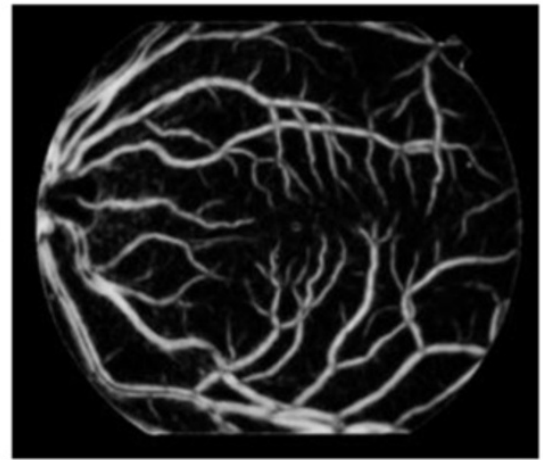

(e)

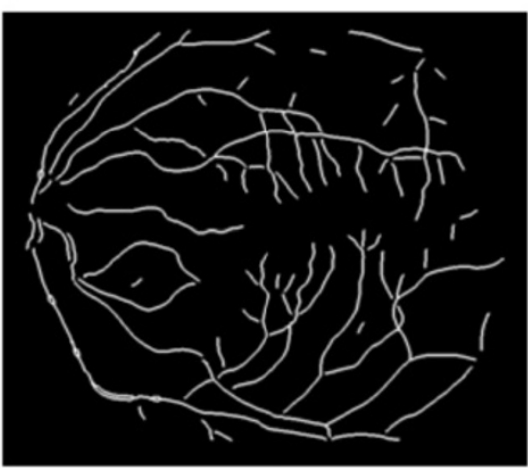

(h)

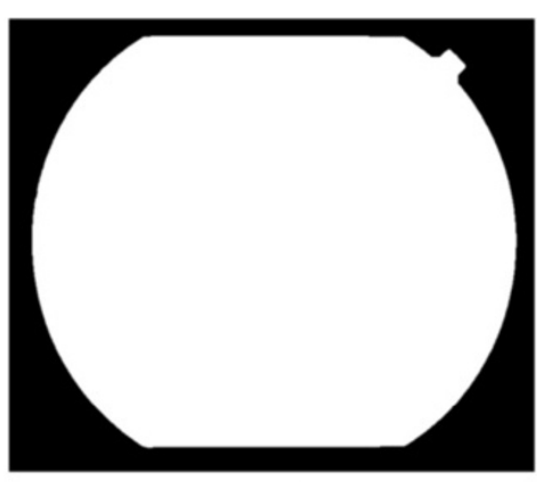

(c)

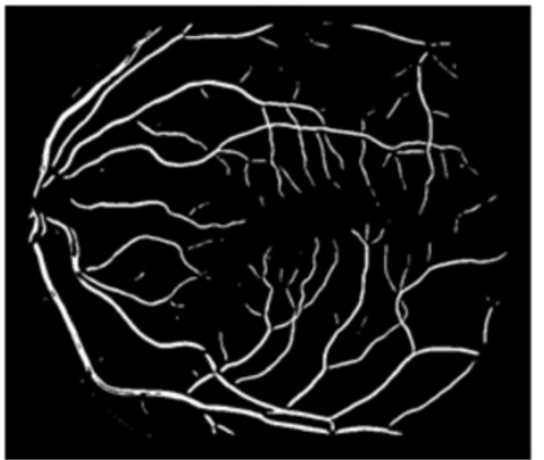

(f)

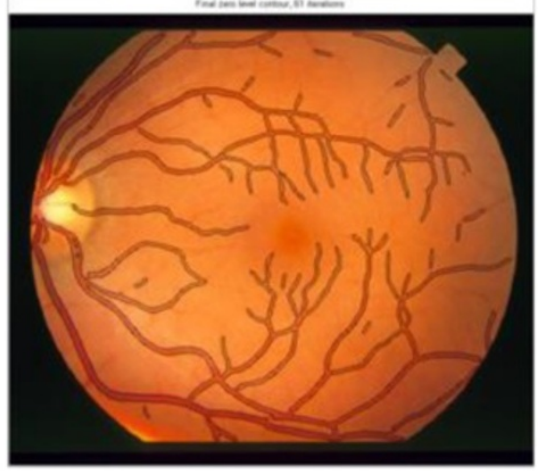

(i)

Figure 17 Segmentation processes of a $700 \times 605$-pixel non-pathological retinal fundus image obtained from STARE dataset. Input image (a), manual segmentation (first observer) (b), mask image (c), image edge obtained using the proposed phase map (d), image structure obtained using the phase map (e), binary image obtained from (d) using Otsu thresholding [43] (f), skeletonized version of (f) after eliminating outliers $(\mathbf{g})$, dilated version of $(\mathbf{g}) \mathbf{( h )}$, segmented image using the proposed method after 61 iterations (i). 
However, some retinal vessels (marked with a blue circle) are still not connected. Therefore, in order to generate a good result as seen in Figure 12f, $\vartheta$ and $\alpha$ are set to 0.6 and 3 , respectively.

Our segmentation process illustrated in Figure 14 employs the skeletonized version of the input image on which a morphological dilation operator is performed only once to initialize the level set function. The proposed method generates good results; some very thin retinal vessels with poor contrast are still missed due to the fact that our method is unable to produce a proper phase map. However, unlike previous works [24,25], the

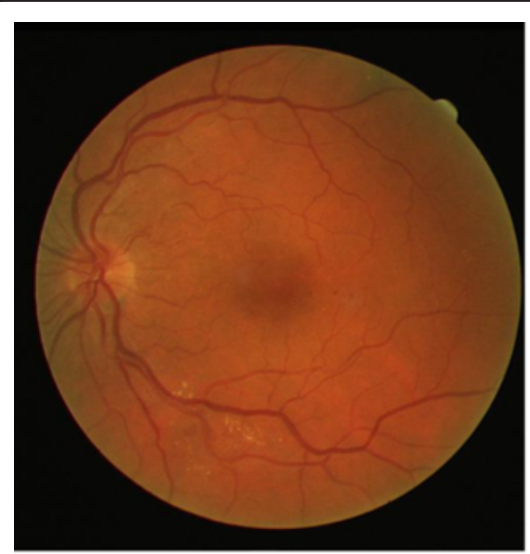

(a)

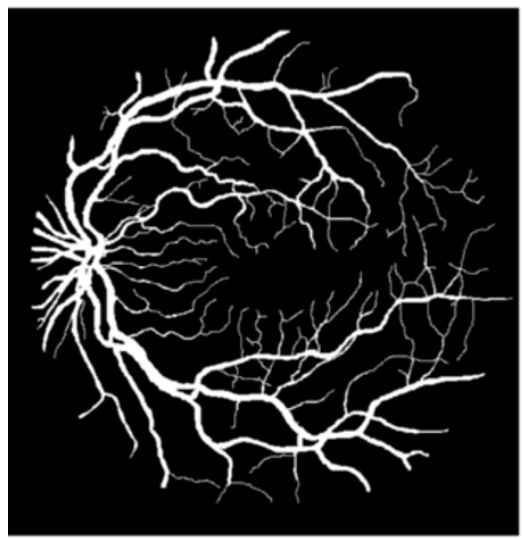

(d)

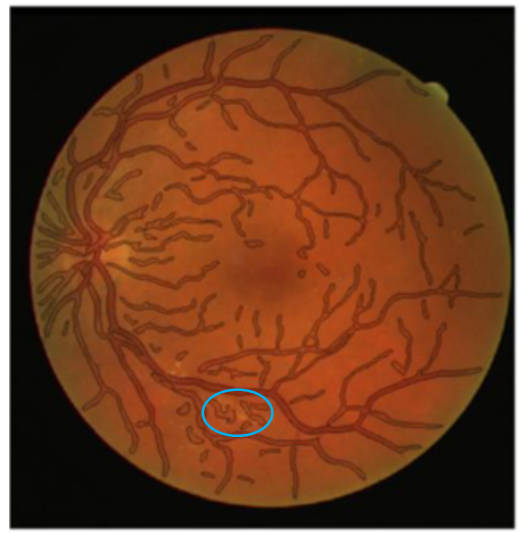

(g)

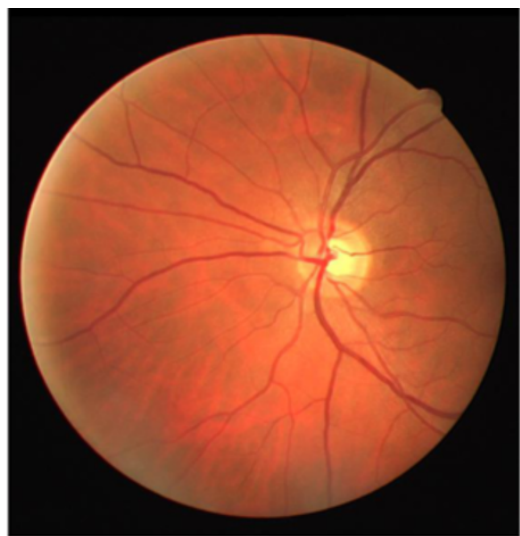

(b)

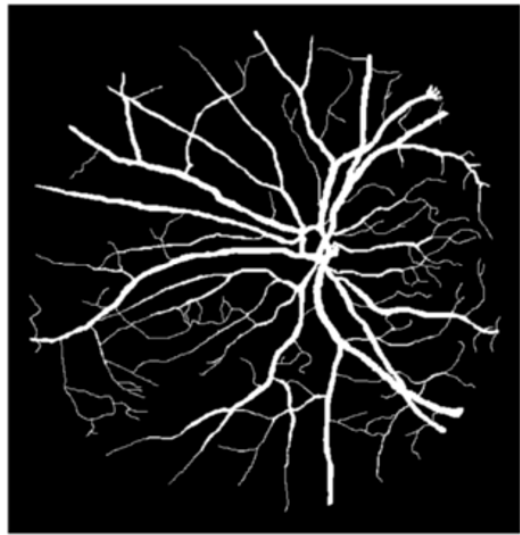

(e)

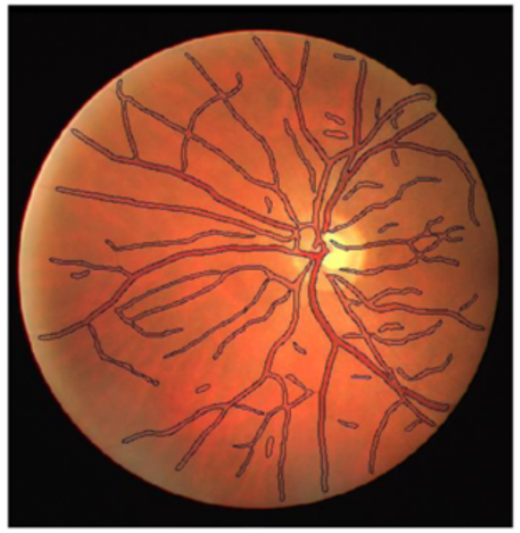

(h)

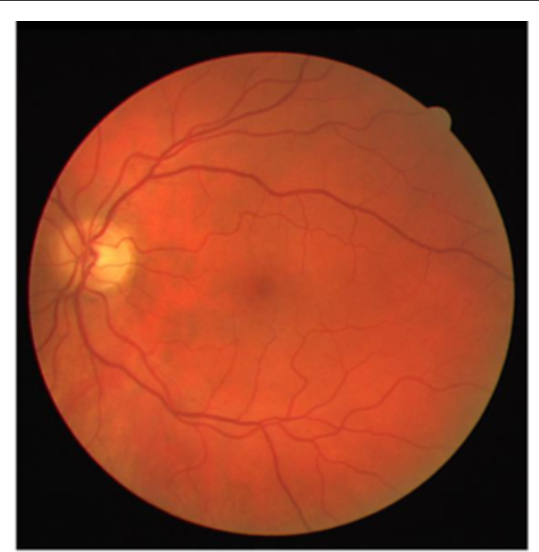

(c)

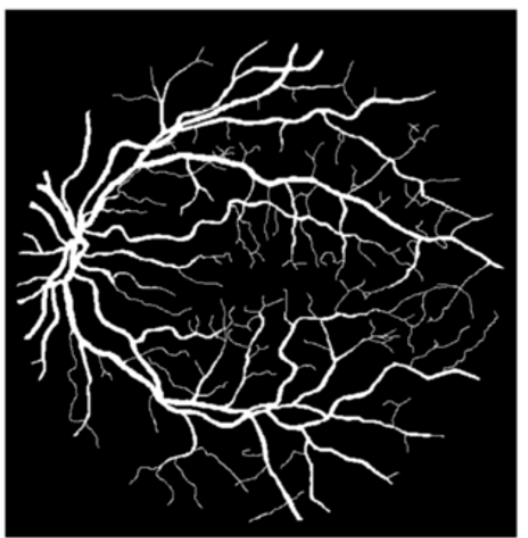

(f)

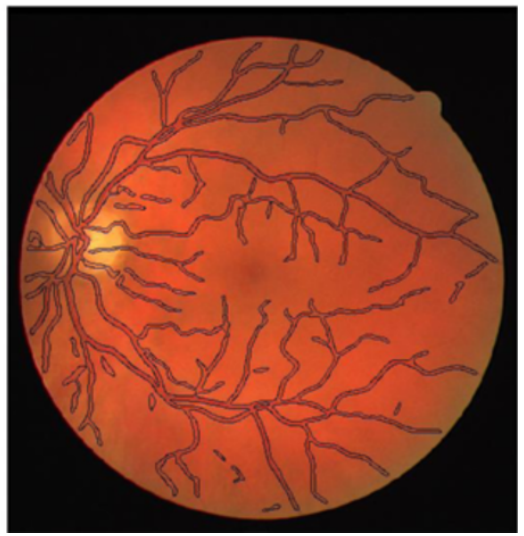

(i)

Figure 18 Vessel segmentation results for $565 \times 584$-pixel pathological and non-pathological images obtained from DRIVE dataset. Pathological image (a), non-pathological image (b), and another non-pathological image (c) (row 1). Manual segmentations of images in row 1 (row 2). Segmented images (row 3). 
method can trace retinal vessels efficiently, since the structure-based level set segmentation method is able to shrink or expand automatically as displayed in Figure 14h where the level set function is initialized inside the vessels in some regions and outside the vessels in some regions.

The test results of preprocessing operations on our dataset are shown Figure 15. The non-uniform intensities in the given image are estimated and corrected properly.

A sample vessel segmentation result for a nonpathological fundus image from our dataset is shown in Figure 16. Here, the approach cannot trace some vessels with poor contrasts as seen in Figure 16h. Also, the image could not be segmented employing the proposed segmentation method without preprocessing as depicted in Figure 16g.
Another sample vessel segmentation result for a nonpathological fundus image from STARE dataset is depicted in Figure 17. Here, the vessels can be traced properly using the method.

The results of other test images in DRIVE, our images, and STARE datasets are given in Figures 18, 19 and 20. Some segmentation results for pathological images include artifact, and they are marked with blue circles. These regions have also poor contrast, and retinal vessels in these regions are very thin.

Figure 21 depicts another case, for which both methods described in earlier work [24,25] failed especially at regions with poor contrast. However, the proposed method is able to properly track the vessels in those regions as shown in Figure 21e. Although the

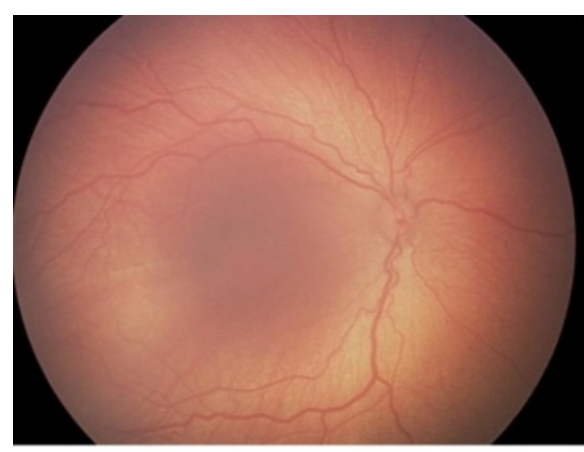

(a)

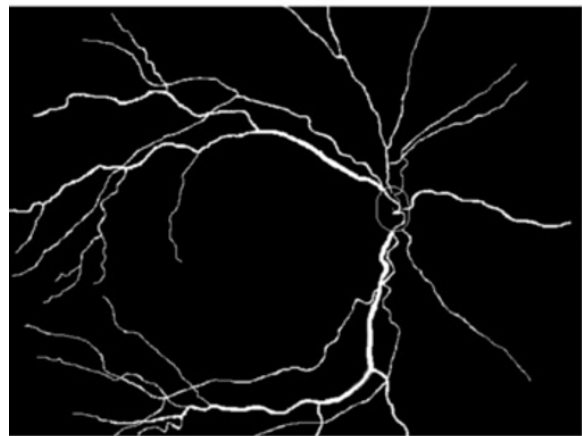

(c)

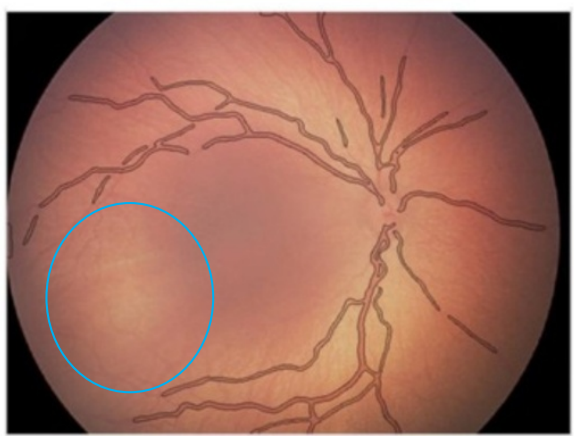

(e)

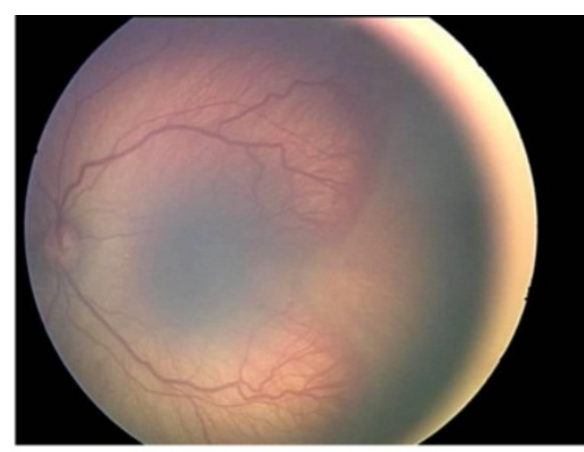

(b)

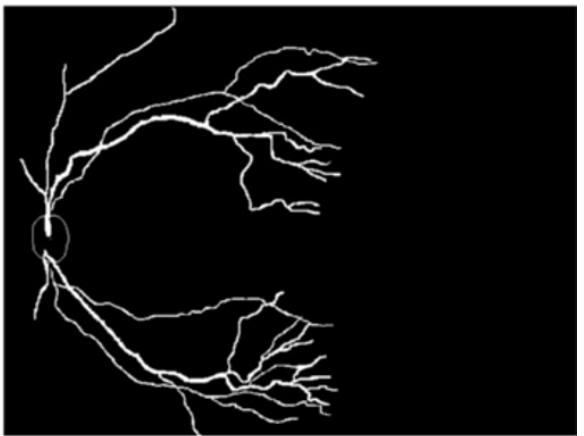

(d)

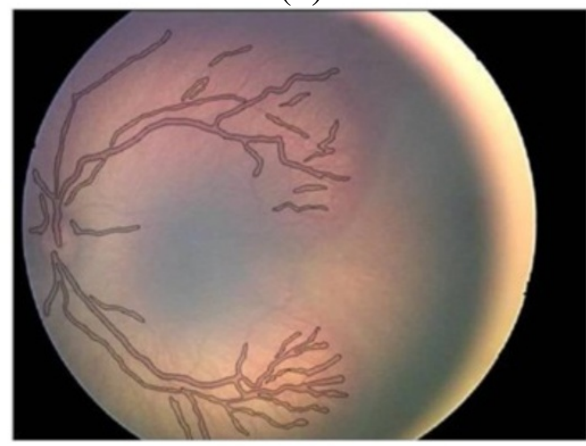

(f)

Figure 19 Vessel segmentation results for $640 \times 480$-pixel non-pathological and pathological images obtained from our dataset. Non-pathological image (a), and pathological image (b) (row 1). Manual segmentations of images in row 1 (row 2). Segmented images (row 3). 
PBLS method presented in [24] runs faster than ours since it employs a narrow band implementation, that faster method is unable to trace retinal vessels properly due to the fact that the phase map of the method is not estimated correctly in the regions with thin vessels and poor contrasts. Also, the DRLSE method proposed in [25] does not expand the vessels if the initialization starts inside the vessel. On the other hand, if initialization starts outside the vessel and the image is in poor contrast, the DRLSE method over-segments the vessels because it uses the image gradient instead of the phase map. Instead of a TV approach, $\partial \Phi_{L} / \partial t=\vartheta \delta_{\varepsilon}(\Phi)$ $\operatorname{div}\left(g \frac{\nabla \Phi}{\|\nabla \Phi\|}\right)$, if an oriented Laplacian flow approach, $\partial \Phi_{L} / \partial t=\vartheta \delta_{\varepsilon}(\Phi)\left(\Phi_{\zeta \zeta}+g \Phi_{\eta \eta}\right)$, proposed in our work, is employed in the DRLSE method to smooth zero-level contours, the results may visually seem to be like oversmoothing as displayed in Figure 21b,c. But, as depicted

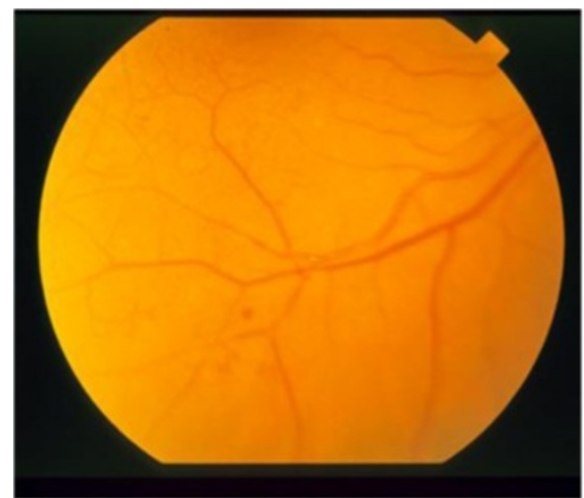

(a)

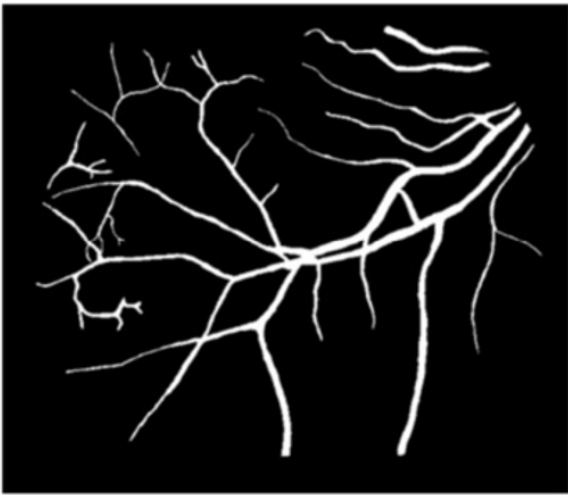

(c)

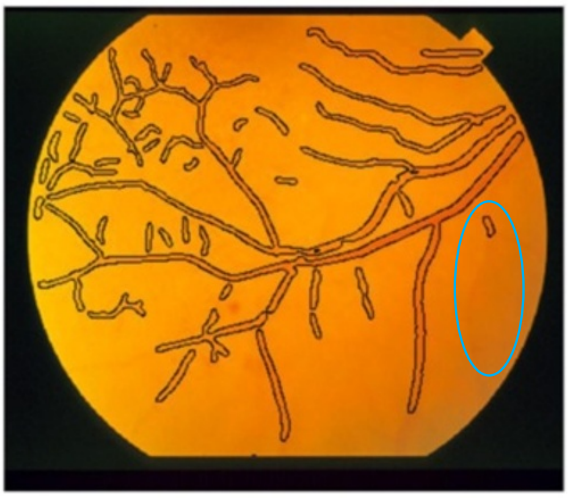

(e)

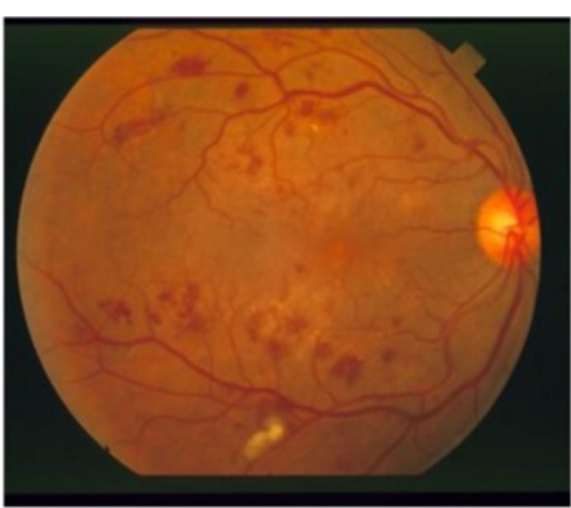

(b)

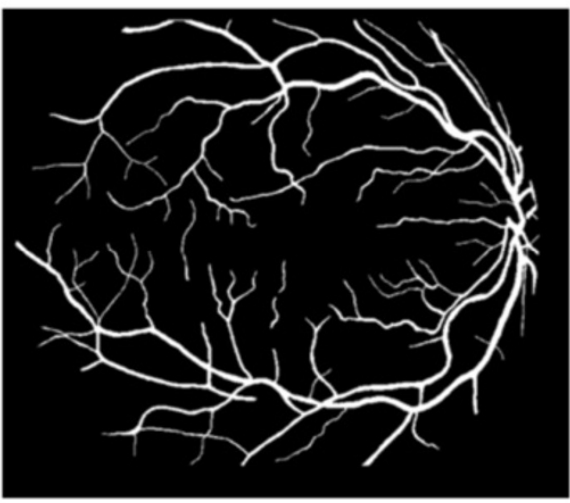

(d)

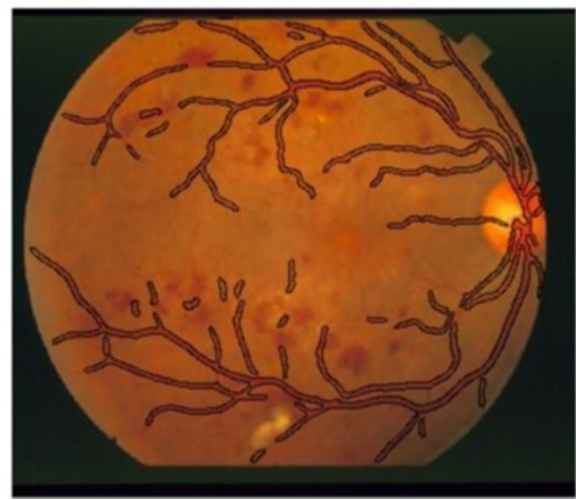

(f)

Figure 20 Vessel segmentation results for $700 \times 605$-pixel non-pathological and pathological images obtained from STARE dataset. Non-pathological image (a), and pathological image (b) (row 1). Manual segmentations of images (first observer) in row 1 (row 2), and segmented images (row 3). 


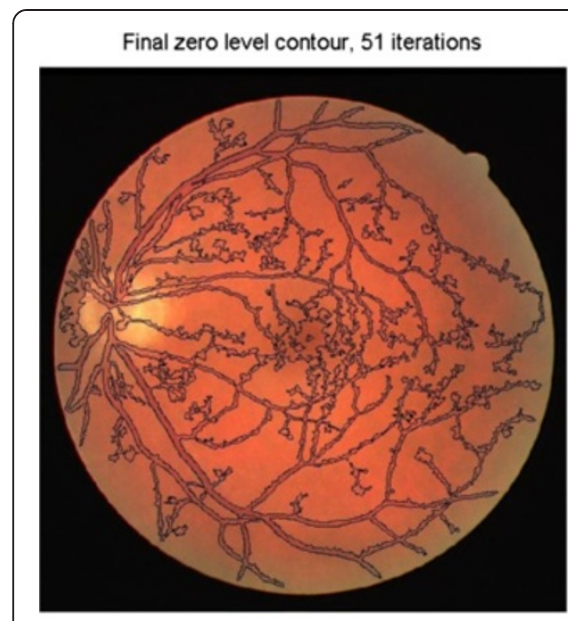

(a)

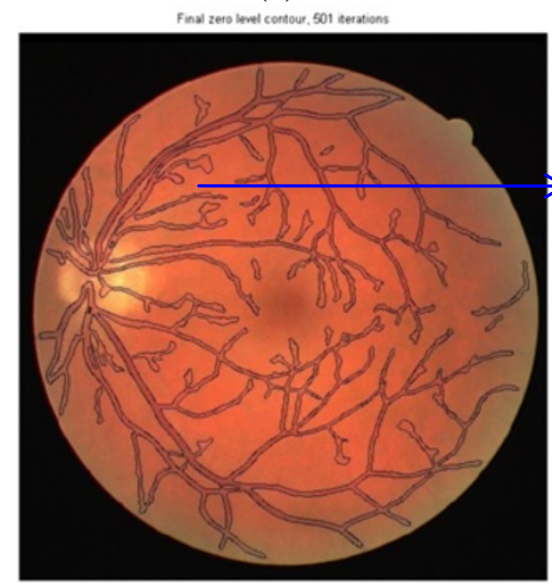

(d)

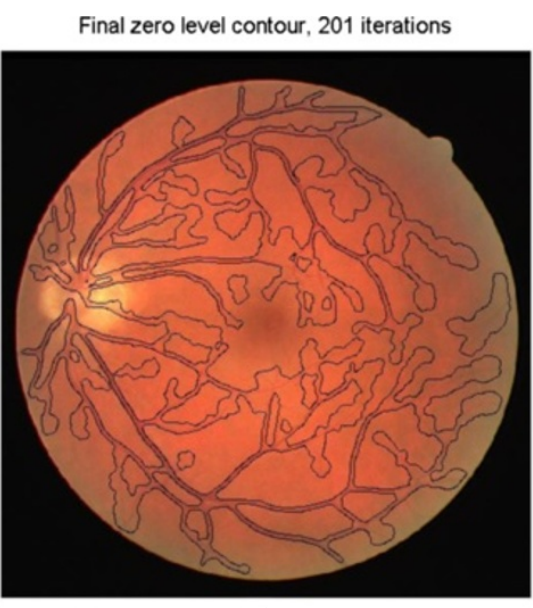

(b)

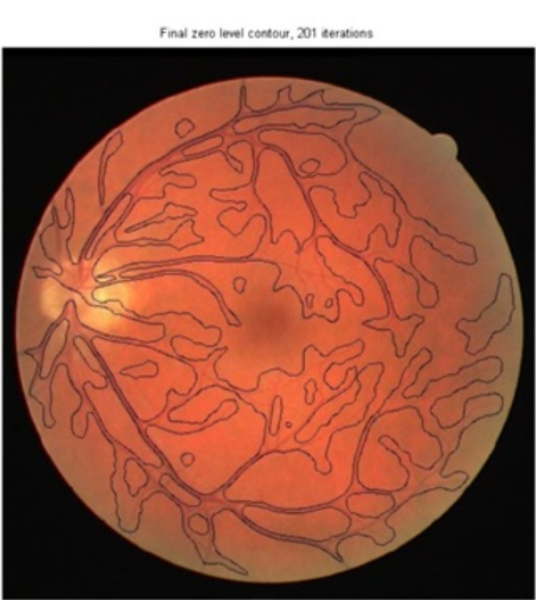

(c)

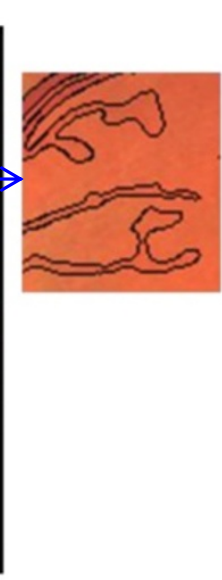

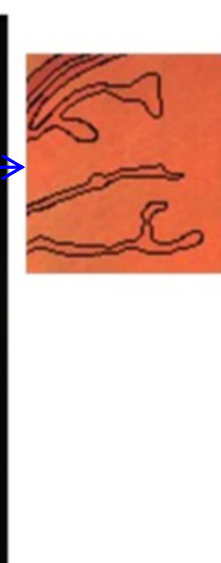

(e)

Figure 21 Vessel segmentation results for the non-pathological fundus image. The PBLS method [24] after 51 iterations (a), the DRLSE method [25] based on TV approach after 201 iterations (b), the DRLSE method [25] based on oriented Laplacian flow approach after 201 iterations (c), the proposed method based on modified TV approach after 501 iterations (d), and the proposed method based on modified oriented Laplacian flow approach after 501 iterations (e).

in Figure 21e, this disadvantage turns into an advantage if the modified oriented Laplacian flow approach, $\partial \Phi_{L} / \partial$ $t=9 \delta_{\varepsilon}(\Phi)\left(\Phi_{\eta \eta}+s(\|\hat{q}\|) \Phi_{\eta \eta}\right)$, is employed in our method, since it eliminates the expansion of segmented vessel areas. As shown in Figure 21d, the expansion is not completely eliminated if a modified TV approach, $\partial$ $\Phi_{L} / \partial t=9 \delta_{\varepsilon}(\Phi) \operatorname{div}(s(\|\hat{q}\|) \nabla \Phi /\|\nabla \Phi\|)$, is used in our method. Also, the vessels could be traced more properly in the proposed method, if the iteration number is increased, but this increases the cost. The result of our method seems to be more efficient compared to the existing methods $[24,25]$ in the literature since it has a novel zero-level contour regularization term and it employs a modified phase map.

Lastly, the segmentation results of the non-pathological image generated by the region-based level set evolution method (RBLSE) [33] are given in Figure 22. The most important advantage of this method is that the initialization may start on any region of the fundus image instead of around vessels by a simple selection. The segmentation of vessels can be done in the fundus images without poor contrast in the initialization phase. After that, while some segmented vessels are combined, some gradually disappeared in later iterations. But surprisingly, after the 42nd iteration, all segmented vessels are gone and only the boundary of the retina remains as segmented as presented in Figure 22c.

Quantitative results are obtained for both datasets where manual vessel segmentation labeling was performed and verified by medical experts. Comparing the results with manual delineations, we obtain overall statistical quality metrics such as sensitivity Se, specificity 


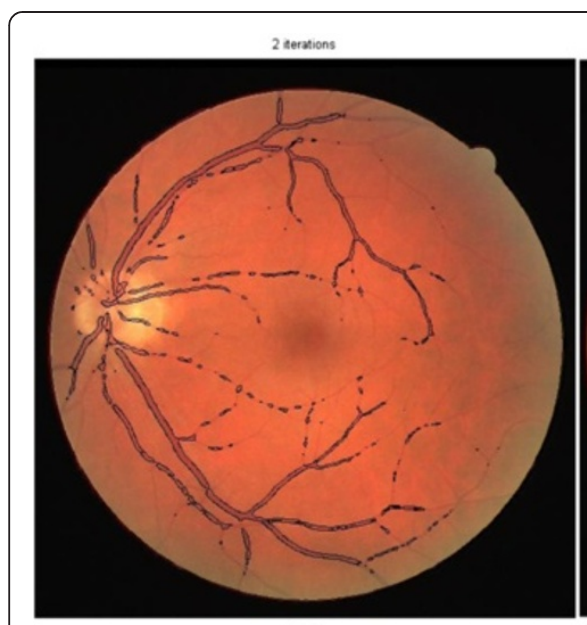

(a)

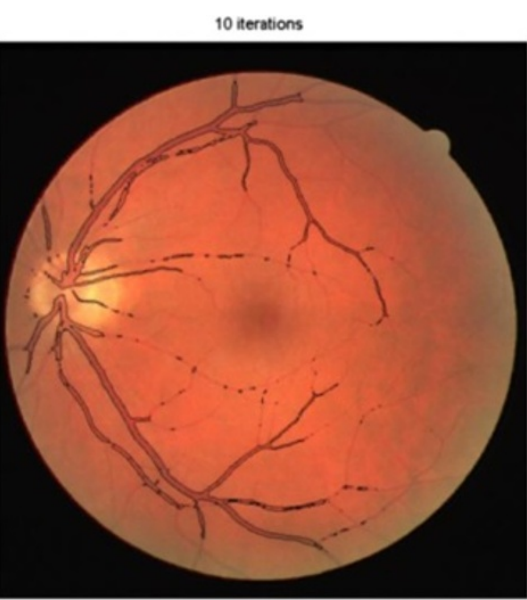

(b)

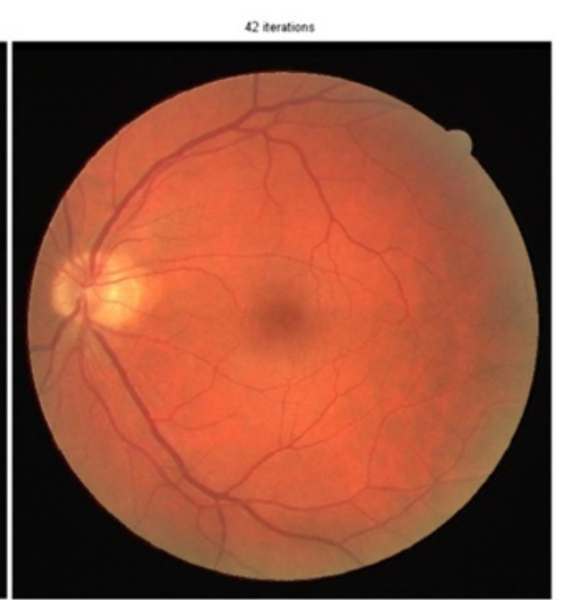

(c)

Figure 22 Vessel segmentation results obtained by the RBLSE method [33] for the non-pathological fundus image. After 2 iterations (a), after 10 iterations (b), and after 42 iterations (c).

$\mathrm{Sp}$, positive predictive value $\mathrm{Ppv}$, negative predictive value Npv, accuracy Acc [14], and kappa $\kappa$ [45]. These measures are given as follows:

$$
\begin{aligned}
& \mathrm{Se}=\frac{\mathrm{TP}}{\mathrm{TP}+\mathrm{FN}}, \quad \mathrm{Sp}=\frac{\mathrm{TN}}{\mathrm{TN}+\mathrm{FP}}, \quad \mathrm{Ppv}=\frac{\mathrm{TP}}{\mathrm{TP}+\mathrm{FP}}, \\
& \mathrm{Npv}=\frac{\mathrm{TN}}{\mathrm{TN}+\mathrm{FN}}, \mathrm{Acc}=\frac{\mathrm{TP}+\mathrm{TN}}{\mathrm{TP}+\mathrm{FP}+\mathrm{TN}+\mathrm{FN}}, \\
& \text { and } \kappa=\frac{2(\mathrm{TP} \times \mathrm{TN}-\mathrm{FP} \times \mathrm{FN})}{(\mathrm{TP}+\mathrm{FP})(\mathrm{FP}+\mathrm{TN})+(\mathrm{TP}+\mathrm{FN})(\mathrm{FN}+\mathrm{TN})} .
\end{aligned}
$$

Here, TP refers to a pixel labeled as vessel by both the algorithm and the medical experts' ground truth data, while TN refers to a pixel that is deemed to be nonvessel by both. FN refers to pixels of vessels (according to ground truth data) missed by the algorithm, and FP refers to pixels falsely categorized by the algorithm as vessel. In order to compare the proposed method, the same statistical metrics for supervised and unsupervised methods [11,14-17] on DRIVE dataset, our dataset, and STARE dataset are also reported in Tables 3, 4, 5, and 6. Here, it should be addressed that, although vascular segmentation has been achieved in countless studies, some of which even have better results in the literature, it has not been done so far using structure-based level set approach. In addition, for instance, while the unsupervised method [17] has good accuracy metric results, it generates occasional artifacts, such as false vessels, next to the optic disks. The results of our method are promising due to the fact that the method does not use any training algorithm compared to the supervised methods presented in [14-17]. As can be seen in Table 6 from the
Acc and $K$ metrics, for instance, when compared with PBLS method, our method fairs better quantitatively.

The methods are implemented using MATLAB R2010a. The programs are executed on a laptop with a Pentium 2.20-GHz processor and a 2-GB RAM. The segmentation of the retinal fundus image with a size of $565 \times 584$ pixels, as depicted in Figure 12f, lasts 61 iterations and $92.69 \mathrm{~s}$. Note that the run time of the program may vary according to structure and size of the retinal fundus image.

\section{Conclusions}

We present a structure-based level set method with automatic seed point selection for segmentation of retinal vasculature in fundus images. Extensive experiments employing the proposed algorithms using datasets indicate that the algorithm performs well and favorably compared to the already existing level set-based methods in the literature. Developing strategies to improve inconsistencies in clinical diagnosis is an important challenge in ophthalmology. The segmentation methods described in this study may provide a basis for the development of computer-based image analysis algorithms. Future work will involve quantitative feature extraction from segmented retinal vessels, followed by implementation of these image analysis algorithms for image-based diagnostic assistance.

We plan to extend the study in order to improve the results especially for pathological regions such as drusen, GA, etc. Moreover, we will investigate how to use all color channels of the given image interactively in an efficient manner in order to trace retinal vasculature more properly. In addition to this, we plan to do a narrow band implementation in order to accelerate the run time of the proposed method. 
Table 3 Statistical results of our method for test images of 1 to 20 from DRIVE dataset

\begin{tabular}{|c|c|c|c|c|c|c|c|}
\hline Dataset & Image number & Se & Sp & Ppv & Npv & Acc & $\kappa$ \\
\hline \multirow[t]{20}{*}{ DRIVE } & 1 & 0.8182 & 0.9581 & 0.7461 & 0.9723 & 0.9398 & 0.7457 \\
\hline & 2 & 0.7764 & 0.9654 & 0.7982 & 0.9608 & 0.9371 & 0.7502 \\
\hline & 3 & 0.7387 & 0.9513 & 0.7218 & 0.9551 & 0.9202 & 0.6834 \\
\hline & 4 & 0.7456 & 0.9677 & 0.7826 & 0.9607 & 0.9378 & 0.7279 \\
\hline & 5 & 0.7419 & 0.9682 & 0.7878 & 0.9593 & 0.9371 & 0.7279 \\
\hline & 6 & 0.7142 & 0.9726 & 0.8126 & 0.9534 & 0.9358 & 0.7233 \\
\hline & 7 & 0.7507 & 0.9466 & 0.6846 & 0.9609 & 0.9204 & 0.6699 \\
\hline & 8 & 0.7285 & 0.9619 & 0.7332 & 0.9610 & 0.9325 & 0.6923 \\
\hline & 9 & 0.7223 & 0.9738 & 0.7880 & 0.9630 & 0.9439 & 0.7221 \\
\hline & 10 & 0.7535 & 0.9656 & 0.7507 & 0.9661 & 0.9400 & 0.7179 \\
\hline & 11 & 0.7456 & 0.9512 & 0.6972 & 0.9613 & 0.9243 & 0.6768 \\
\hline & 12 & 0.7932 & 0.9594 & 0.7391 & 0.9697 & 0.9383 & 0.7297 \\
\hline & 13 & 0.7165 & 0.9665 & 0.7811 & 0.9533 & 0.9307 & 0.7073 \\
\hline & 14 & 0.7940 & 0.9573 & 0.7130 & 0.9720 & 0.9380 & 0.7160 \\
\hline & 15 & 0.7867 & 0.9463 & 0.6321 & 0.9742 & 0.9296 & 0.6616 \\
\hline & 16 & 0.7880 & 0.9697 & 0.7989 & 0.9677 & 0.9457 & 0.7621 \\
\hline & 17 & 0.7581 & 0.9715 & 0.7900 & 0.9660 & 0.9451 & 0.7425 \\
\hline & 18 & 0.8407 & 0.9517 & 0.6965 & 0.9784 & 0.9388 & 0.7271 \\
\hline & 19 & 0.8696 & 0.9599 & 0.7504 & 0.9815 & 0.9489 & 0.7764 \\
\hline & 20 & 0.8254 & 0.9604 & 0.7162 & 0.9785 & 0.9458 & 0.7364 \\
\hline
\end{tabular}

Table 4 Statistical results of our method for test images of 1 to 20 from our dataset

\begin{tabular}{|c|c|c|c|c|c|c|c|}
\hline Dataset & Image number & Se & Sp & Ppv & Npv & Acc & $\kappa$ \\
\hline \multirow[t]{20}{*}{ Ours } & 1 & 0.4821 & 0.9905 & 0.7483 & 0.9702 & 0.9623 & 0.5676 \\
\hline & 2 & 0.3471 & 0.9887 & 0.6680 & 0.9586 & 0.9494 & 0.4330 \\
\hline & 3 & 0.5745 & 0.9786 & 0.5802 & 0.9781 & 0.9589 & 0.5557 \\
\hline & 4 & 0.5617 & 0.9695 & 0.5911 & 0.9657 & 0.9398 & 0.5437 \\
\hline & 5 & 0.5699 & 0.9872 & 0.7537 & 0.9708 & 0.9602 & 0.6284 \\
\hline & 6 & 0.3527 & 0.9879 & 0.6416 & 0.9613 & 0.9511 & 0.4318 \\
\hline & 7 & 0.8109 & 0.9566 & 0.5234 & 0.9885 & 0.9485 & 0.6098 \\
\hline & 8 & 0.3134 & 0.9887 & 0.6280 & 0.9594 & 0.9499 & 0.3950 \\
\hline & 9 & 0.3966 & 0.9857 & 0.5666 & 0.9719 & 0.9591 & 0.4461 \\
\hline & 10 & 0.4290 & 0.9878 & 0.5958 & 0.9764 & 0.9654 & 0.4814 \\
\hline & 11 & 0.2789 & 0.9901 & 0.6119 & 0.9607 & 0.9523 & 0.3619 \\
\hline & 12 & 0.5497 & 0.9840 & 0.6101 & 0.9796 & 0.9651 & 0.5602 \\
\hline & 13 & 0.6942 & 0.9783 & 0.6056 & 0.9852 & 0.9653 & 0.6288 \\
\hline & 14 & 0.4028 & 0.9844 & 0.5128 & 0.9759 & 0.9616 & 0.4316 \\
\hline & 15 & 0.5848 & 0.9849 & 0.5977 & 0.9840 & 0.9700 & 0.5756 \\
\hline & 16 & 0.3300 & 0.9877 & 0.6573 & 0.9539 & 0.9440 & 0.4133 \\
\hline & 17 & 0.7426 & 0.9731 & 0.5785 & 0.9870 & 0.9622 & 0.6308 \\
\hline & 18 & 0.5550 & 0.9762 & 0.5149 & 0.9797 & 0.9578 & 0.5122 \\
\hline & 19 & 0.7238 & 0.9712 & 0.6101 & 0.9826 & 0.9567 & 0.6392 \\
\hline & 20 & 0.6576 & 0.9680 & 0.4891 & 0.9838 & 0.9542 & 0.5373 \\
\hline
\end{tabular}


Table 5 Statistical results of our method for test images of 1 to 20 from STARE dataset

\begin{tabular}{|c|c|c|c|c|c|c|c|}
\hline Dataset & Image number & Se & Sp & Ppv & Npv & Acc & $\kappa$ \\
\hline \multirow[t]{20}{*}{ STARE } & 1 & 0.6449 & 0.9731 & 0.7455 & 0.9574 & 0.9374 & 0.6570 \\
\hline & 2 & 0.5754 & 0.9836 & 0.7795 & 0.9584 & 0.9464 & 0.6336 \\
\hline & 3 & 0.8036 & 0.9519 & 0.5973 & 0.9820 & 0.9398 & 0.6527 \\
\hline & 4 & 0.3117 & 0.9972 & 0.9275 & 0.9271 & 0.9271 & 0.4376 \\
\hline & 5 & 0.8084 & 0.9466 & 0.6803 & 0.9723 & 0.9296 & 0.6985 \\
\hline & 6 & 0.7759 & 0.9666 & 0.6912 & 0.9781 & 0.9498 & 0.7035 \\
\hline & 7 & 0.8567 & 0.9631 & 0.7412 & 0.9820 & 0.9514 & 0.7674 \\
\hline & 8 & 0.7758 & 0.9644 & 0.7125 & 0.9742 & 0.9451 & 0.7121 \\
\hline & 9 & 0.7814 & 0.9698 & 0.7569 & 0.9736 & 0.9495 & 0.7406 \\
\hline & 10 & 0.7568 & 0.9722 & 0.7711 & 0.9700 & 0.9485 & 0.7349 \\
\hline & 11 & 0.8000 & 0.9629 & 0.7004 & 0.9780 & 0.9470 & 0.7175 \\
\hline & 12 & 0.8446 & 0.9665 & 0.7490 & 0.9814 & 0.9537 & 0.7679 \\
\hline & 13 & 0.7743 & 0.9710 & 0.7881 & 0.9687 & 0.9470 & 0.7510 \\
\hline & 14 & 0.7611 & 0.9739 & 0.8055 & 0.9663 & 0.9474 & 0.7528 \\
\hline & 15 & 0.6239 & 0.9796 & 0.8031 & 0.9512 & 0.9376 & 0.6680 \\
\hline & 16 & 0.4445 & 0.9916 & 0.8961 & 0.9165 & 0.9150 & 0.5528 \\
\hline & 17 & 0.7238 & 0.9803 & 0.8372 & 0.9621 & 0.9488 & 0.7477 \\
\hline & 18 & 0.5669 & 0.9931 & 0.8594 & 0.9685 & 0.9635 & 0.6647 \\
\hline & 19 & 0.4183 & 0.9934 & 0.8001 & 0.9646 & 0.9595 & 0.5304 \\
\hline & 20 & 0.8035 & 0.9512 & 0.6234 & 0.9797 & 0.9377 & 0.6679 \\
\hline
\end{tabular}

Table 6 Statistical average results for test images of 1 to 20 from the datasets

\begin{tabular}{|c|c|c|c|c|c|c|c|c|}
\hline \multirow{2}{*}{$\begin{array}{l}\text { Dataset } \\
\text { DRIVE }\end{array}$} & \multicolumn{2}{|r|}{ Method } & \multirow{2}{*}{$\begin{array}{c}\text { Se } \\
0.7754\end{array}$} & \multirow{2}{*}{$\begin{array}{c}\text { Sp } \\
0.9348\end{array}$} & \multirow{2}{*}{$\begin{array}{c}\text { Ppv } \\
0.6403\end{array}$} & \multirow{2}{*}{$\begin{array}{c}\text { Npv } \\
0.9655\end{array}$} & \multirow{2}{*}{$\begin{array}{c}\text { Acc } \\
0.9140\end{array}$} & \multirow{2}{*}{$\begin{array}{c}\boldsymbol{\kappa} \\
0.6494\end{array}$} \\
\hline & Unsupervised & PBLS [24] & & & & & & \\
\hline & & Jiang et al. [12] & - & - & - & - & 0.9212 & - \\
\hline & & Martinez-Perez et al. [13] & 0.7246 & 0.9655 & - & - & 0.9344 & - \\
\hline & & Proposed & 0.7704 & 0.9613 & 0.7460 & 0.9658 & 0.9365 & 0.7198 \\
\hline & & Budai et al. [17] & 0.6440 & 0.9870 & - & - & 0.9572 & - \\
\hline & Supervised & Staal et al. [16] & 0.7194 & 0.9773 & - & - & 0.9442 & - \\
\hline & & Marin et al. [14] & 0.7067 & 0.9801 & 0.8433 & 0.9582 & 0.9452 & - \\
\hline & & Soares et al. [15] & 0.7283 & 0.9788 & - & - & 0.9466 & - \\
\hline \multirow[t]{2}{*}{ Ours } & Unsupervised & PBLS [24] & 0.6600 & 0.9482 & 0.4380 & 0.9804 & 0.9328 & 0.4754 \\
\hline & & Proposed & 0.5179 & 0.9810 & 0.6042 & 0.9737 & 0.9567 & 0.5192 \\
\hline \multirow[t]{7}{*}{ STARE } & Unsupervised & PBLS [24] & 0.8268 & 0.9117 & 0.5227 & 0.9803 & 0.9035 & 0.5822 \\
\hline & & Hoover et al. [11] & 0.6751 & 0.9567 & - & - & 0.9267 & - \\
\hline & & Martinez-Perez et al. [13] & 0.7506 & 0.9569 & - & - & 0.9410 & - \\
\hline & & Proposed & 0.6926 & 0.9726 & 0.7633 & 0.9656 & 0.9441 & 0.6779 \\
\hline & Supervised & Soares et al. [15] & 0.7103 & 0.9737 & - & - & 0.9480 & - \\
\hline & & Staal et al. [16] & 0.6970 & 0.9810 & - & - & 0.9516 & - \\
\hline & & Marin et al. [14] & 0.6944 & 0.9819 & - & - & 0.9526 & - \\
\hline
\end{tabular}




\section{Competing interests}

The authors declare that they have no competing interests.

\section{Acknowledgements}

This work is partially supported by grants from TUBITAK (grant no. 1059B191000548), NSF, and NIH.

\section{Author details}

${ }^{1}$ Department of Computer Engineering, Karadeniz Technical University, Trabzon 61080, Turkey. ${ }^{2}$ Cognitive Systems Laboratory, Northeastern University, Boston, MA 02115, USA. ${ }^{3}$ Martinos Imaging Center, Massachusetts General Hospital, Boston, MA 02129, USA. ${ }^{4}$ Department of Ophthalmology, Oregon Health \& Science University, Portland, OR 97239-3098, USA. ${ }^{5}$ Department of Medical Informatics, Oregon Health \& Science University, Portland, OR 97239-3098, USA.

\section{Received: 27 February 2014 Accepted: 30 July 2014} Published: 11 August 2014

\section{References}

1. MF Chiang, L Jiang, R Gelman, YE Du, JT Flynn, Interexpert agreement of plus disease diagnosis in retinopathy of prematurity. Arch. Ophthalmol $125,875-880$ (2007)

2. R Gelman, L Jiang, YE Du, ME Martinez-Perez, JT Flynn, MF Chiang, Plus disease in retinopathy of prematurity: pilot study of computer-based and expert diagnosis. JAAPOS 11(6), 532-540 (2007)

3. A Osareh, B Shadgar, An automated tracking approach for extraction of retinal vasculature in fundus images. J. Opthalmic. Vis. Res. 5, 20-26 (2010)

4. D Wu, M Zhang, JC Liu, W Bauman, On the adaptive detection of blood vessels in retinal images. IEEE Trans. Biomed. Eng. 53, 341-343 (2006)

5. MZC Azemin, DK Kumar, TY Wong, R Kawasaki, P Mitchell, JJ Wang, Robust methodology for fractal analysis of the retinal vasculature. IEEE Trans. Med. Imaging 2(30), 243-250 (2011)

6. V Mahadevan, H Narasimha-lyer, B Roysam, HL Tanenbaum, Robust modelbased vasculature detection in noisy biomedical images. IEEE Trans. Inf. Technol. Biomed. 8(3), 360-376 (2004)

7. H Narasimha-lyer, V Mahadevan, JM Beach, B Roysam, Improved detection of the central reflex in retinal vessels using a generalized dual-Gaussian model and robust hypothesis testing. IEEE Trans. Inf. Technol. Biomed. 3(12), 406-410 (2008)

8. KW Tobin, E Chaum, VP Govindasamy, TP Karnowski, Detection of anatomic structures in human retinal imagery. IEEE Trans. Med. Imaging 26(12), 1729-1739 (2007)

9. M Niemeijer, X Xu, AV Dumitrescu, P Gupta, B van Ginneken, JC Folk, MD Abramoff, Automated measurement of the arteriolar-to-venular width ratio in digital color fundus photographs. IEEE Trans. Med. Imaging 11(30), 1941-1950 (2011)

10. L Wang, A Bhalerao, R Wilson, Analysis of retinal vasculature using a multiresolution Hermite model. IEEE Trans. Med. Imaging 2(26), 137-152 (2007)

11. A Hoover, $V$ Kouznetsova, M Goldbaum, Locating blood vessels in retinal images by piecewise threshold probing of a matched filter response. IEEE Trans. Med. Imaging 19(3), 203-210 (2000)

12. X Jiang, D Mojon, Adaptive local thresholding by verification-based multithreshold probing with application to vessel detection in retinal images. IEEE Trans. Pattern. Anal. Mach. Intell. 25(1), 131-137 (2003)

13. ME Martinez-Perez, AD Hughes, SA Thom, AA Bharath, KH Parker, Segmentation of blood vessels from red-free and fluoresce in retinal images. Med. Image Anal. 11(1), 47-61 (2007)

14. D Marin, A Aquino, GME Arias, JM Bravo, A New supervised method for blood vessel segmentation in retinal images by using gray-level and moment invariants-based features. IEEE Trans. Med. Imaging 30(1), 146-158 (2011)

15. JVB Soares, JJG Leandro, RM Jr Cesar, HF Jelinek, MJ Cree, Retinal vessel segmentation using the 2-D Gabor wavelet and supervised classification. IEEE Trans. Med. Imaging 25(9), 1214-1222 (2006)

16. J Staal, MD Abramoff, M Niemeijer, MA Viergever, B van Ginneken, Ridge-based vessel segmentation in color images of the retina. IEEE Trans. Med. Imaging 23, 501-509 (2004)
17. A Budai, R Bock, A Maier, J Hornegger, G Michelson, Robust vessel segmentation in fundus images. Int. J Biomed. Imaging 2013, (2013)

18. AF Frangi, WJ Niessen, KL Vincken, MA Viergever, Multiscale Vessel Enhancement Filtering (Springer (Germany, Heidelberg, 1998)

19. S You, E Bas, D Erdogmus, J Kalpathy-Cramer, Principal curve based retinal vessel segmentation towards diagnosis of retinal diseases. Proc. Healthcare Inform, Imaging Sys. Biol. (HISB) 331-337 (2011). San Jose, California, USA, (2011)

20. D Erdogmus, U Ozertem, Self-consistent locally defined principal surfaces. Proc. ICASSP Vol. 2, II.549-II.552 (2007). Honolulu, Hawaii, USA

21. C Kirbas, F Quek, Vessel Extraction Techniques and Algorithms: a Survey. Proceedings of the Third IEEE Symposium on Biolnformatics and BioEngineering (BIBE'03), 238-245 (Bethesda, Maryland, USA, 2003)

22. L Vese, $T$ Chan, A multiphase level set framework for image segmentation using the Mumford and Shah model. Int. J. Comput. Vis. 50(3), 271-293 (2002)

23. V Caselles, R Kimmel, G Sapiro, Geodesic active contours. Int. J. Comput. Vis. 22(1), 61-79 (1997)

24. G Lathen, J Jonasson, M Borga, Blood vessel segmentation using multi-scale quadrature filtering. Pattern Recogn. Lett. 31, 762-767 (2010)

25. C Li, C Xu, C Gui, MD Fox, Distance regularized level set evolution and its application to image segmentation. IEEE Trans. Image Process. 19(12), 3243-3254 (2010)

26. KY Pang, L Iznita, A Fadzil, AN Hanung, N Hermawan, SA Vijanth, Segmentation of Retinal Vasculature in Colour Fundus Images (Conference on Innovation Technologies in Intelligent Systems and Industrial Applications (CITISIA, Malaysia, 2009), pp. 398-401

27. B Zhou, C Mu, Level set evolution for boundary extraction based on a p-Laplace equation. Appl. Math. Mod 34(12), 3910-3916 (2010)

28. L Meng, $\mathrm{H}$ Chuanjiang, $Z$ Yi, Adaptive regularized level set method for weak boundary object segmentation. Math. Probl. Eng 2012(369472), 16 (2012). doi:10.1155/2012/369472

29. A Belaid, D Boukerroui, Y Maingourd, J-F Lerallut, Phase based level set segmentation of ultrasound images. IEEE Trans. Inform. Tech. Biomed 15(1), 138-147 (2011)

30. B Dizdaroğlu, E Ataer-Cansizoglu, J Kalpathy-Cramer, K Keck, MF Chiang, D Erdogmus, Level Sets for Retinal Vasculature Segmentation Using Seeds from Ridges and Edges from Phase Maps. 2012 IEEE International Workshop On Machine Learning For Signal Processing (Santander, Spain, 2012)

31. G Yu, P Lin, P Li, Z Bian, Region-based vessel segmentation using level set framework. Int. J. Control. Autom. Syst. 4(5), 660-667 (2006)

32. C Li, C Kao, JC Gore, Z Ding, Minimization of region-scalable fitting energy for image segmentation. EEE Trans. Image Proc. 17(10), 1940-1949 (2008)

33. C Li, R Huang, Z Ding, C Gatenby, DN Metaxas, JC Gore, A level set method for image segmentation in the presence of intensity inhomogeneities with application to MRI. IEEE Trans. Image Proc. 20(7), 2007-2016 (2011)

34. YQ Zhao, XH Wang, XF Wang, FY Shih, Retinal vessels segmentation based on level set and region growing. Pattern Recognition. 47(7), 2437-2446 (2014)

35. M Bertalmio, L Vese, G Sapiro, S Osher, Simultaneous structure and texture image inpainting. IEEE Trans. Image Process. 12, 882-889 (2003)

36. A Buades, TM Le, J-M Morel, LA Vese, Fast cartoon + texture image filters. IEEE Trans. Image Process. 19(8), 1978-1986 (2010)

37. B Dizdaroğlu, An image completion method using decomposition. EURASIP J. Advanc. Signal Proc 2011(831724), 15 (2011). doi:10.1155/2011/831724

38. MJ Black, G Sapiro, DH Marimont, D Heeger, Robust anisotropic diffusion. IEEE Trans. Image Process. 7(3), 421-432 (1998)

39. D Tschumperlé, PDE's based regularization of multi-valued images and applications, PhD thesis (Université de Nice-Sophia Antipolis, France, 2002)

40. P Kovesi, Phase congruency: a low-level image invariant. Psychological Research 64(2), 136-148 (2000)

41. P Blomgren, TF Chan, CK Wong, Total variation image restoration: numerical methods and extensions. Proc. IEEE Int. Conf Image Proc 3, 384-387 (1997)

42. P Kornprobst, R Deriche, G Aubert, Image Restoration via PDE's (First Annual Symposium on Enabling Technologies for Law Enforcement and Security SPIE Conference 2942: Investigative Image Processing, Boston, Massachusetts, USA, 1996) 
43. N Otsu, A threshold selection method from gray-level histogram. IEEE Trans. Syst. Man Cybern 9(1), 62-66 (1979)

44. R Courant, K Friedrichs, $\mathrm{H}$ Lewry, Über die partiellen Differenzengleichungen der mathematischen Physik. Math. Ann. 100(1), 32-74 (1928)

45. J Landis, G Koch, The measurement of observer agreement for categorical data. Biometrics 33, 159-174 (1977)

doi:10.1186/1687-5281-2014-39

Cite this article as: Dizdaroğlu et al: Structure-based level set method for automatic retinal vasculature segmentation. EURASIP Journal on Image and Video Processing 2014 2014:39.

Submit your manuscript to a SpringerOpen ${ }^{\circ}$ journal and benefit from:

- Convenient online submission

Rigorous peer review

- Immediate publication on acceptance

- Open access: articles freely available online

- High visibility within the field

- Retaining the copyright to your article

Submit your next manuscript at springeropen.com 